\title{
DEGENERATE BIFURCATIONS NEAR A DOUBLE EIGENVALUE IN THE BRUSSELATOR
}

\author{
GERHARD DANGELMAYR ${ }^{1}$
}

(Received 12 January 1984; revised 2 December 1985)

\begin{abstract}
The steady state bifurcations near a double zero eigenvalue of the reaction diffusion equation associated with a tri-molecular chemical reaction (the Brusselator) are analysed. Special emphasis is put on three degeneracies where previous results of Schaeffer and Golubitsky do not apply. For these degeneracies it is shown by means of a LiapunovSchmidt reduction that the steady state bifurcations are determined by codimension-three normal forms. They are of types $(9)_{31},(8)_{221}$ and $(6 a)_{\rho, \kappa}$ in a recent classification of $Z$ (2)-equivariant imperfect bifurcations with corank two. Each normal form couples an ordinary corank-1 bifurcation in the sense of Golubitsky and Schaeffer to a degenerate $Z$ (2)-equivariant corank-1 bifurcation of Golubitsky and Langford in a specific way.
\end{abstract}

\section{Introduction}

The reaction-diffusion equations for the tri-molecular chemical model of Levefer and Prigogine [10] - meanwhile well known as the "Brusselator-model"- serve as a laboratory for various bifurcation phenomena leading to pattern formation, that is, bifurcation from a spatially and temporally homogeneous solution (the trivial solution) to several types of inhomogeneous solutions depending on the range of parameters $[1,2,8-11]$. Here we are interested in the formation of steady state spatially inhomogeneous solutions resulting from a double zero eigenvalue. Several authors $[2,8,10]$ have discussed the case of a simple eigenvalue and first attempts towards an understanding of the non-simple case have been made by Keener [9]. A detailed study near a double eigenvalue has been performed by Schaeffer and Golubitsky [11] by using the general theory of imperfect bifurcations via singularity theory developed in $[5,6]$.

\footnotetext{
${ }^{1}$ Institute for Information Science, University of Tübingen, Tübingen, Federal Republic of Germany. (c) Copyright Australian Mathematical Society 1987, Serial-fee code 0334-2700/87
} 
The approach of [11] is essentially the following. By means of a LiapunovSchmidt reduction a set of two bifurcation equations was derived for two bifurcation variables-the "amplitudes" of the "bifurcating modes"-and a distinguished bifurcation parameter. Then it was shown that the reduced bifurcation equations are contact equivalent (in the sense of singularity theory) to a certain normal form of codimension two which, by unfolding, reveals all possible bifurcation diagrams in the vicinity of the bifurcation point. Since the original model-equations possess a natural reflection symmetry, the reduced bifurcation equations are equivariant under the action of the symmetry group $Z(2)$ and the unfolding has to respect this symmetry. The results in [11] are valid provided some certain nondegeneracy conditions are satisfied by the parameters.

There are four free parameters in the model, but two of them are fixed by the requirement of a double eigenvalue. The space of the remaining two free parameters is divided into several open regions where specific types of bifurcation diagrams result from the normal form. On the boundary between two adjacent regions one of the non-degeneracy conditions is not satisfied.

Our objective is to analyse the bifurcation phenomena in the Brusselator near the boundary separating two adjacent regions. The strategy is similar to that of [11]; that is, a Liapunov-Schmidt reduction yields reduced bifurcation equations which turn out to be contact equivalent to certain normal forms. These normal forms occur in a classification of $Z$ (2)-equivariant imperfect bifurcations carried out in [4]. However, due to the higher degeneracy on a boundary, our normal forms have codimension three and contain those of [11] as subordinate bifurcations. By unfolding and analysing the perturbed bifurcation diagrams associated with a codimension-three normal form, we can move parameters from one region into the adjacent region. This does not only reproduce the diagrams of [11], but also shows how the local branches in each region are connected when crossing the boundary; that is, first attempts are made towards an understanding of the global bifurcation geometry.

A crucial role for the bifurcation analysis is played by the theory of imperfect bifurcations $[5,6]$ which is based on singularity theory. Suitable coordinate transformations allow us to transform the reduced bifurcation equations resulting from the Liapunov-Schmidt reduction described before to certain polynomial normal forms. A classification of these normal forms has been accomplished in [4]. The codimension-three normal forms relevant for the Brusselator are of types $(9)_{31},(8)_{221}$ and $(6 a)_{\rho, k}$ in the classification of [4] whereas the generic normal form of [11] is denoted by $(6)_{\rho}$. The bifurcation geometry for some of the codimension-three normal forms of [4] is discussed in [1] in the context of coupled Hopf and steady state bifurcations. A further objective of this paper is to complement the analyses of [1] by a detailed discussion of the types $(9)_{31},(8)_{221}$ and $(6 a)_{\rho, \kappa}$. Each of these types is divided into several subtypes according to 
different signs and modal regions of the normal form. The analyses presented here contain all cases which appear in the Brusselator and, for completeness, some further cases which are not present in the Brusselator but may occur generically in other model-equations that depend on three or more parameters.

The paper is organized as follows. In Section 1 general nonlinear operator equations with reflection symmetry are considered and a perturbation procedure is developed for the Liapunov-Schmidt reduction. In Section 2 the model equations are introduced and cast into a form to which the methods of Section 1 apply. Normal forms which govern the codimension-three degeneracies described before are set up in Section 3. It has been shown in [11] that four different degeneracies occur, but no normal forms have been established. The LiapunovSchmidt reductions required for three of these degeneracies (the types $(9)_{31},(8)_{221}$ and $\left.(6 a)_{\rho, \kappa}\right)$ are carried out in Sections 4-6. In Section 7 the concepts of universal unfoldings and subordinate bifurcations are introduced which are useful tools in the analysis of the bifurcation geometry. Structurally stable bifurcation diagrams associated with those cases of the normal forms $(9)_{31},(8)_{221}$ and $(6 a)_{\rho, \kappa}$ that occur in the Brusselator are analyzed in Sections 8, 9 and 10, respectively. Some other cases corresponding to the types $(8)_{221}$ and $(6 a)_{\rho, \kappa}$ and leading to isola formation are discussed in Section 11. In Section 12 we illustrate how the generic type (6) ${ }_{\rho}$ of [11] is organized by unfolding the various codimension-three degeneracies.

\section{A sample new global result}

In order to exemplify the global results obtained in this paper we describe here some features of the normal form (9) $)_{31}$ and its relation to the analysis of [11]. If $x$ and $y$ are, respectively, the amplitudes of the even and odd unstable modes, then the reduced bifurcation equations have the form $\left(a\left(x, y^{2}, \lambda\right), y b\left(x, y^{2}, \lambda\right)\right)=$ $(0,0)$, that is, the bifurcation diagrams have a reflection symmetry with respect to the plane $y=0$. Here, $\lambda$ is the bifurcation parameter with scale chosen such that the degeneracy occurs at $\lambda=0$. In [11] it has been shown that two adjacent regions of the parameter space of the Brusselator-equations give rise to degenerate bifurcation diagrams which correspond to subtypes of $(6)_{\rho}$ and are denoted by $\mathrm{III}_{0}$ and $\mathrm{III}_{1}$, respectively. They are sketched in Fig. $\mathrm{A}$ where the signs in the brackets indicate the signs of the real parts of the eigenvalues of $d_{(x, y)}(a, y b)$ along a solution branch. The latter $T$ refers to the trivial solution $x=y=0$ corresponding to a spatially homogeneous solution of the model equations. Observe that for both $\mathrm{III}_{0}$ and $\mathrm{III}_{1}$ three branches of solutions bifurcate from the trivial solution at the degenerate bifurcation point, one lying in the plane $y=0$ (symmetric branch) and the others having $y \neq 0$ (asymmetric branches). For III $_{1}$ 
only a subbranch of the symmetric branch is stable whereas no stable nontrivial branch exists for III $_{0}$. The result of Section 4 implies that on the boundary between the regions corresponding to $\mathrm{III}_{0}$ and $\mathrm{III}_{1}$ a codimension-3 degeneracy of type (9) ${ }_{31}$ appears with degenerate bifurcation diagram as shown in Fig. B(a). Here the branches in the plane of symmetry form a pitchfork bifurcation. In addition to the symmetric branches two further asymmetric branches branch off
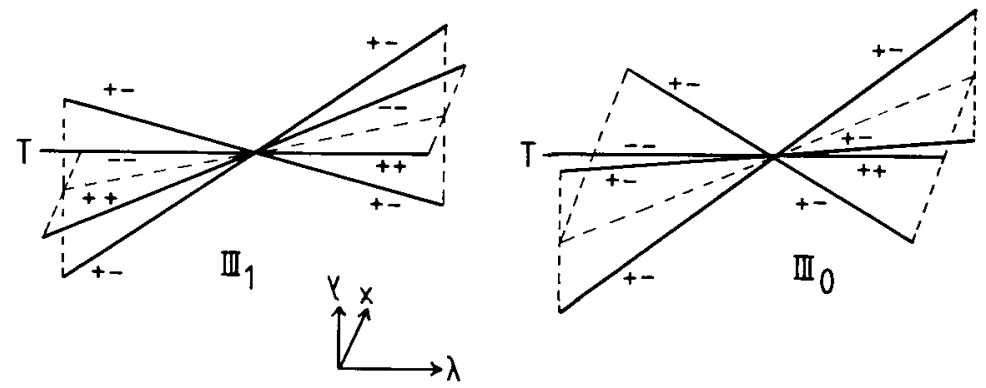

Figure A
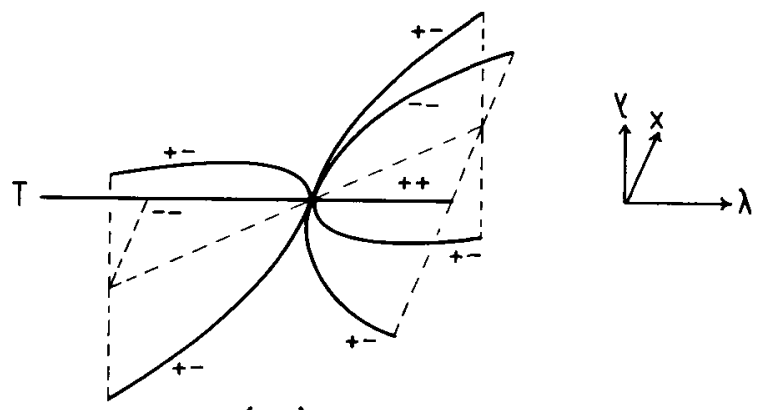

(a)
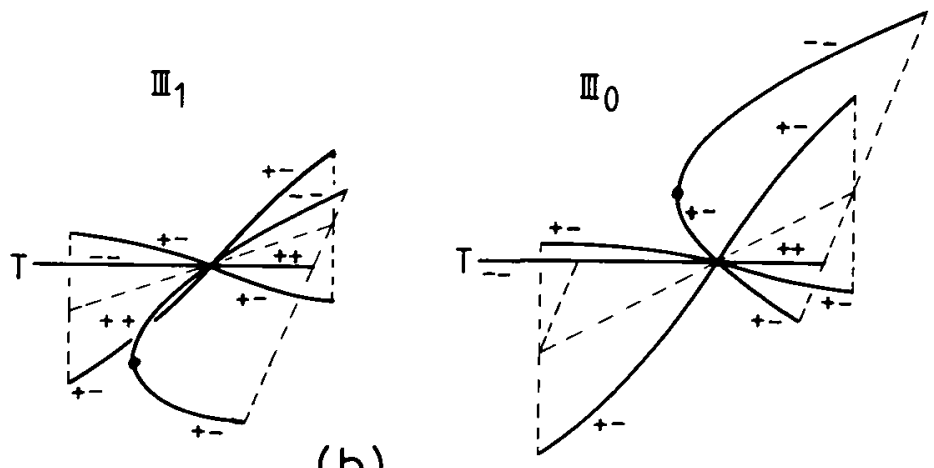

(b)

Figure B 


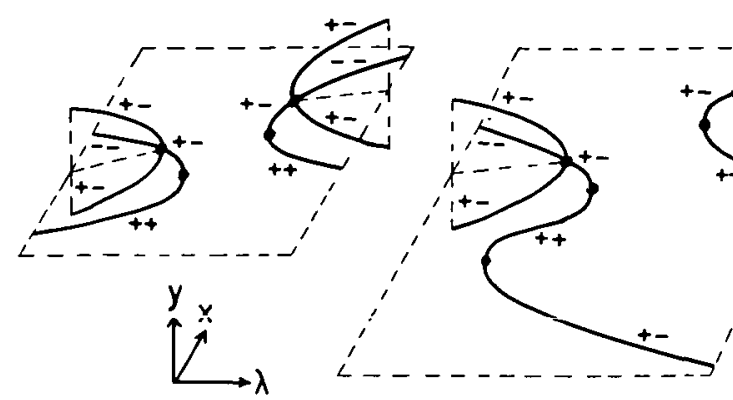

(a) (b)

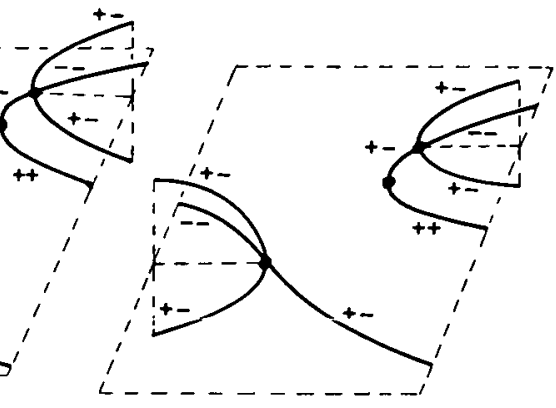

(c)

Figure C

the degenerate bifurcation point. When moving away from the boundary into the regions leading to types $\mathrm{III}_{1}$ or $\mathrm{III}_{0}$ the degeneracy of Fig. $\mathrm{B}(\mathrm{a})$ is removed and the bifurcation diagrams evolve to those shown in Fig. $\mathrm{B}(\mathrm{b})$. This corresponds to a partial unfolding of the normal form (9) ${ }_{31}$, that is, only one of the three unfolding parameters is varied. In a small neighborhood of the degenerate bifurcation points of the diagrams in Fig. B(b) we discover qualitatively the same behaviour of the branches as in Fig. A. The globalization of the local results of [11] consists in the appearance of limit points in the symmetric branches. Similar globalizations are obtained by unfolding the other codimension-3 degeneracies (see Section 12).

The structurally stable (or perturbed) bifurcation diagrams in a neighborhood of a codimension- 2 degeneracy of [11] (e.g. III $_{1}$ ) are determined by the full unfolding of the underlying normal form and the same holds for the codimension-3 degeneracies considered in this paper. Each of the former diagrams can be discovered as part of a structurally stable diagram of one of the codimension-3 cases. In Fig. C(a) we have sketched a structurally stable bifurcation diagram corresponding to the case $\mathrm{III}_{1}$ which is part of the diagram of Fig. $\mathrm{C}(\mathrm{b})$ corresponding to type (9) ${ }_{31}$. Contrary to that the diagram of Fig. C(c), also corresponding to type (9) ${ }_{31}$, does not contain any of the perturbed bifurcation diagrams obtained in [11] as part of it. This demonstrates that globalizations as well as new diagrams are obtained by analyzing the codimension- 3 degeneracies.

\section{Liapunov-Schmidt reduction for general equations}

In this section we recall the basic facts about the Liapunov-Schmidt reduction method for nonlinear operator equations with reflection symmetry. This method will be used in Sections 2, 4-6. 


\subsection{The bifurcation problem}

Consider an equation of the form

$$
F(u, \lambda)=0,
$$

where $F$ is a smooth map from a neighborhood of the origin in $X_{1} \times \mathbb{R}$ to $X$ with $X_{1}, X$ being real Banach spaces. For simplicity it is assumed that $X_{1}$ is dense in $X$ and

$$
F(0, \lambda)=0 \text {. }
$$

The Frechet-derivative with respect to $u \in X_{1}$ and $\lambda \in \mathbb{R}$ is denoted by $d_{u}$ and $d_{\lambda}$, respectively. We make the following assumptions on $F$.

(I) There exists a reflection $R: X \rightarrow X, R^{2}=$ id which commutes with $F$,

$$
F(R u, \lambda)=R F(u, \lambda) \text {. }
$$

Clearly, in order that (1.3) makes sense we must assume that $X_{1}$ is invariant under the action of $R$.

(II) The linearized operator

$$
L=d_{u} F(0,0)
$$

has a two-dimensional null space $N=\operatorname{span}\left\{\phi_{1}, \phi_{2}\right\} \subset X_{1}$. Moreover, $\phi_{1}$ is even and $\phi_{2}$ is odd with respect to $R$,

$$
R \phi_{1}=\phi_{1}, \quad R \phi_{2}=-\phi_{2} .
$$

(III) A Fredholm alternative is valid for $L$ : There exist $\phi_{1}^{*}, \phi_{2}^{*}$ in the dual $X^{*}$ of $X$ such that the equation $L u=v$ is solvable if and only if $\left\langle v, \phi_{1}^{*}\right\rangle=\left\langle v, \phi_{2}^{*}\right\rangle$ $=0$.

(IV) All generalized eigenvectors of $L$ (viewed as densely defined operator in $X$ ) corresponding to the eigenvalue zero belong to $N\left(\operatorname{ker} L^{2}=\operatorname{ker} L\right)$.

Assumptions (III), (IV) imply that we can normalize the $\phi_{i}^{*}$ such that

$$
R^{*} \phi_{i}^{*}=(-1)^{i-1} \phi_{i}^{*} \text { and }\left\langle\phi_{i}, \phi_{j}^{*}\right\rangle=\delta_{i j}(i=1,2),
$$

$\delta_{i}$, being the Kronecker symbol. Assumptions (II)-(IV) are standard in bifurcation theory $[3,12]$ and allow the application of the method of Liapunov and Schmidt which reduces (1.1) to a system of two real equations.

We briefly recall the general Liapunov-Schmidt method. Introduce projections $P, Q$ in $X_{1}$

$$
P u=\sum_{i=1}^{2}\left\langle u, \phi_{i}^{*}\right\rangle \phi_{i}, Q=\text { id }-P
$$


and decompose $X$ into $X=E \oplus N$ where $E=Q X$. Similarly, $X_{1}$ is decomposed into $X_{1}=E_{1} \oplus N$ with $E_{1}=Q X_{1}=E \cap X_{1}$. For the solutions of (1.1) one makes the ansatz $u=x \phi_{1}+y \phi_{2}+w$ where $(x, y) \in \mathbb{R}^{2}$ are coordinates in $N$ and $w \in E_{1}$. Standard arguments of bifurcation theory $[3,11,12]$ show that the equation

$$
Q F\left(x \phi_{1}+y \phi_{2}+w, \lambda\right)=0
$$

has locally a unique and smooth solution $w(x, y, \lambda)$ which satisfies $w(0,0, \lambda)=0$ and contains no linear terms in $(x, y, \lambda)$. Hence, the solutions of (1.1) are uniquely determined by the solutions of the following system of two equations,

$$
G(x, y, \lambda)=\left(\begin{array}{c}
\left\langle F\left(x \phi_{1}+y \phi_{2}+w(x, y, \lambda), \lambda\right), \phi_{1}^{*}\right\rangle \\
\left\langle F\left(x \phi_{1}+y \phi_{2}+w(x, y, \lambda), \lambda\right), \phi_{2}^{*}\right\rangle
\end{array}\right)=0 .
$$

Moreover, the symmetry and (1.5) imply (see [11])

$$
R w(x, y, \lambda)=w(x,-y, \lambda)
$$

and that $G$ has the form

$$
G(x, y, \lambda)=\left(\begin{array}{c}
a(x, \lambda, z) \\
y b(x, \lambda, z)
\end{array}\right), \quad z=y^{2},
$$

with smooth functions $a$ and $b$ satisfying

$$
a(0, \lambda, 0)=0, \quad b(0)=a_{x}(0)=0 .
$$

Clearly, $G$ is equivariant under the $Z(2)$-action $(x, y) \rightarrow(x,-y)$.

\subsection{Computation of Taylor-coefficients}

We give expressions for those Taylor-coefficients

$$
a_{i j k}=\frac{1}{i ! j ! k !} \frac{\partial^{i+j+k} a(0)}{\partial x^{i} \partial \lambda^{\jmath} \partial z^{k}}, \quad b_{i j k}=\frac{1}{i ! j ! k !} \frac{\partial^{t+\jmath+k} b(0)}{\partial x^{i} \partial \lambda^{\jmath} \partial z^{k}}
$$

of $a$ and $b$ which are needed in Sections 2, 4-6. To this end we introduce the multi-linear operators

$$
F_{n m}=\frac{1}{n ! m !} d_{u}^{n} d_{\lambda}^{m} F(0,0): X_{1}^{n} \rightarrow X .
$$

For convenience we use the notation

$$
F_{n m}\left(u_{1}, \ldots, u_{1}, \ldots, u_{l} \cdots u_{l}\right)=F_{n m} u_{1}^{n_{1}} \cdots u_{l}^{n_{l}}
$$

if $u_{i} \in X_{1} \quad(1 \leqslant i \leqslant l)$ occurs $n_{i}$-times on the left-hand side of (1.13) and $n_{1}+\cdots+n_{l}=n$. We also need the operator

$$
L_{0}=\left.Q L\right|_{E_{1}}: E_{1} \rightarrow E
$$

which, in virtue of assumptions (III), (IV), has a bounded inverse. 
The solution $w$ of (1.7) is split into even and odd parts,

$$
w(x, y, \lambda)=V(x, \lambda, z)+y U(x, \lambda, z)
$$

where $R V=V$ and $R U=-U$. The Taylor coefficients of $U$ are denoted by

$$
U_{i j k}=\frac{1}{i ! j ! k !} \frac{\partial^{i+j+k} U(0)}{\partial x^{\imath} \partial \lambda^{j} \partial z^{k}} \in E_{1}
$$

and analogously $V_{i j k}$. We can express the coefficients of $U$ and $V$ in the form

$$
U_{i j k}=-L_{0}^{-1} Q B_{i j k}, \quad V_{i j k}=-L_{0}^{-1} Q A_{i j k},
$$

where $B_{\imath j k}$ and $A_{\imath \jmath k}$ are vectors in $E$ and $A_{100}=A_{0 n 0}=0$. Those of lowest order (quadratic in $(x, y, \lambda)$ ) are given by

$$
\begin{array}{lll}
A_{110}=F_{11} \phi_{1}, & B_{010}=F_{11} \phi_{2}, & \\
A_{200}=F_{20} \phi_{1}^{2}, & A_{001}=F_{20} \phi_{2}^{2}, & B_{100}=2 F_{20} \phi_{1} \phi_{2} .
\end{array}
$$

From (1.17) and (1.18) we can compute the following vectors (cubic in $(x, y, \lambda)$ ),

$$
\begin{aligned}
& A_{300}=2 F_{20} \phi_{1} V_{200}+F_{30} \phi_{1}^{3} \\
& A_{101}=2 F_{20}\left(\phi_{2} U_{100}+\phi_{1} V_{001}\right)+3 F_{30} \phi_{1} \phi_{2}^{2} \\
& A_{011}=2 F_{20} \phi_{2} U_{010}+F_{11} V_{001}+F_{21} \phi_{2}^{2} \\
& B_{200}=2 F_{20}\left(\phi_{2} V_{200}+\phi_{1} U_{100}\right)+3 F_{30} \phi_{1}^{2} \phi_{2} \\
& B_{001}=2 F_{20} \phi_{2} V_{001}+F_{30} \phi_{2}^{3} .
\end{aligned}
$$

Using the last equation of (1.19) in the first equation of (1.17) allows us to compute $U_{001}$ from which the only "fourth order vector" (needed in Section 6) is obtained,

$$
A_{002}=F_{20}\left(V_{001}^{2}+2 \phi_{2} U_{001}\right)+3 F_{30} \phi_{2}^{2} V_{001}+F_{40} \phi_{2}^{4} .
$$

The Taylor coefficients of $a$ and $b$ required in Sections 2, 4-6 are then computed from (1.18)-(1.20) via

$$
a_{i j k}=\left\langle A_{i j k}, \phi_{1}^{*}\right\rangle, \quad b_{\imath j k}=\left\langle B_{i j k}, \phi_{2}^{*}\right\rangle .
$$

\section{Formulation of the problem}

\subsection{The model equations}

The relevant equations for the Brusselator model are [11]

$$
\frac{\partial u}{\partial t}=L u+N(u), \quad u=\left(\begin{array}{l}
U \\
V
\end{array}\right)
$$

where

$$
L=\left(\begin{array}{cc}
D_{1} & 0 \\
0 & D_{2}
\end{array}\right) \frac{\partial^{2}}{\partial \xi^{2}}+\left(\begin{array}{cc}
B-1 & A^{2} \\
-B & -A^{2}
\end{array}\right)
$$




$$
N(u)=\left(\frac{B}{A} U^{2}+2 A U V+U^{2} V\right)\left(\begin{array}{r}
1 \\
-1
\end{array}\right) \text {. }
$$

Equation (2.1) is a parabolic system for the unknown functions $U(\xi), V(\xi)$ where $\xi \in[0, \pi]$, subject to Dirichlet-boundary conditions

$$
U(0)=U(\pi)=V(0)=V(\pi)=0 .
$$

Here, $D_{1}$ and $D_{2}$ are diffusion coefficients, $A, B$ are externally controlled concentrations and $U$ and $V$ describe deviations of the concentrations of two chemical reactants from spatially independent steady values $A$ and $B / A$, respectively.

The following notation will be used throughout the paper,

$$
\theta=D_{2} / D_{1}, \quad D=D_{1} .
$$

We regard $L$ as a linear operator acting on, say, $C^{0}\left([0, \pi], \mathbb{R}^{2}\right)$ with homogeneous Dirichlet boundary conditions. The eigenfunctions of $L$ have the form

$$
\left(\begin{array}{l}
a_{l \pm} \\
b_{l \pm}
\end{array}\right) \sin l \xi, \quad l \in N
$$

with eigenvalues $\kappa_{l_{ \pm}}$, where $\kappa_{l_{ \pm}}$and $\left(a_{l_{ \pm}}, b_{l_{ \pm}}\right)$are eigenvalues and eigenvectors of the matrix

$$
M_{l}=\left(\begin{array}{cc}
B-1-l^{2} D & A^{2} \\
-B & -A^{2}-l^{2} D \theta
\end{array}\right) .
$$

The parameter $B$ is considered as a distinguished bifurcation parameter, that is, we look for bifurcations of new solutions of (2.1) from the trivial solution $u=0$ as $B$ is increased. Both time independent and time periodic solutions can bifurcate from the trivial solution, but we consider only the former case. For coupled bifurcations of steady state and time periodic modes see [1].

In [11] it has been shown that the first bifurcation is from a double zero eigenvalue of $L$ if and only if

$$
A=A_{k} \equiv \sqrt{\theta \mu_{1} \mu_{2}} D \quad \text { and } \quad B=B_{k} \equiv\left(1+D \mu_{1}\right)\left(1+D \mu_{2}\right),
$$

where

$$
\mu_{1}=k^{2}, \quad \mu_{2}=(k+1)^{2},
$$

holds for some integer $k$. If $(2.7)$ is satisfied, then the zero eigenfunctions are [11]

$$
\phi_{1}=\left(\begin{array}{c}
\theta D \mu_{1} \\
-1-D \mu_{1}
\end{array}\right) \sin k \xi, \quad \phi_{2}=\left(\begin{array}{c}
\theta D \mu_{2} \\
-1-D \mu_{2}
\end{array}\right) \sin (k+1) \xi
$$


We also need the eigenfunctions $\phi_{1}^{*}, \phi_{2}^{*}$ corresponding to the zero eigenvalue of the adjoint $L^{*}$ of $L$,

$$
\phi_{1}^{*}=f_{1}\left(\begin{array}{c}
1+D \mu_{2} \\
D \mu_{2}
\end{array}\right) \sin k \xi, \quad \phi_{2}^{*}=f_{2}\left(\begin{array}{c}
1+D \mu_{1} \\
D \mu_{1}
\end{array}\right) \sin (k+1) \xi,
$$

where the normalization constants

$$
1 / f_{1}=\frac{\pi}{2} D\left\{\theta \mu_{\imath}-\mu_{J}+D \mu_{1} \mu_{2}(\theta-1)\right\}, \quad i, j=1,2, i \neq j
$$

are chosen in such a way that $\left(\phi_{i}, \phi_{j}^{*}\right)=\delta_{i}$, with $(\cdot, \cdot)$ denoting the $L^{2}$-inner product.

Since (2.1) does not depend explicitly on $\xi$, the problem possesses a natural reflection symmetry, namely, the right-hand side of (2.1) commutes with $R$ where

$$
R u(\xi)=u(\pi-\xi) \text {. }
$$

Observing that

$$
R \sin l \xi=(-1)^{l+1} \sin l \xi
$$

there is always one odd and one even zero eigenfunction of $L$. Assume that the double degeneracy occurs for some $j \in N$, that is, $\kappa_{j-}=\kappa_{j+1-}=0$. Following [11] we use (2.7)-(2.11) with $k=j$ if $j$ is odd and with $k=-(j+1)$ if $j$ is even. In this way it is achieved that $\phi_{1}, \phi_{1}^{*}$ are even and $\phi_{2}, \phi_{2}^{*}$ are odd with respect to the symmetry (2.12).

\subsection{The unperturbed problem}

Following [11] we define now the unperturbed problem for (2.1). To this end the Banach spaces $X=C^{0}\left([0, \pi], \mathbb{R}^{2}\right)$ and $X_{1}=\left\{u \in C^{2}\left([0, \pi], \mathbb{R}^{2}\right): u(0)=\right.$ $u(\pi)=0\}$ are introduced. For $A=A_{k}$ and fixed $D, \theta$, the right-hand side of (2.1) induces a smooth map $F: X_{1} \times \mathbb{R} \rightarrow X$ via $F(u, \lambda)=L u+N(u)$ where we have set

$$
\lambda=B-B_{k} .
$$

The unperturbed problem is then defined by the equation

$$
F(u, \lambda)=0 \text {. }
$$

The steady states of (2.1) are determined by (2.14).

In order to apply the method of Section 1 to (2.14) we have to check the assumptions (I)-(IV). Clearly, assumptions (I)-(III) hold, however, assumption (IV) is only satisfied if $1 / f_{1} f_{2} \neq 0$ which will be assumed throughout the paper. In Subsection 2.5 we shall see that stability of the bifurcating solutions requires the further restriction that $f_{1}$ and $f_{2}$ must be positive. 
We give now the expressions for the multi-linear operators $F_{n m}$, Eq. (1.12), to be used in Sections 4-6:

$$
\begin{aligned}
& F_{20} u_{1} u_{2}=\left(1 / D \sqrt{\theta \mu_{1} \mu_{2}}\right)\left\{B_{k} U_{1} U_{2}+D^{2} \theta \mu_{1} \mu_{2}\left(U_{1} V_{2}+U_{2} V_{1}\right)\right\}\left(\begin{array}{r}
1 \\
-1
\end{array}\right) \\
& F_{11} u=U\left(\begin{array}{r}
1 \\
-1
\end{array}\right), \quad F_{21} u_{1} u_{2}=\left(1 / D \sqrt{\theta \mu_{1} \mu_{2}}\right) U_{1} U_{2}\left(\begin{array}{r}
1 \\
-1
\end{array}\right) \\
& \Gamma_{30} \ddot{u}_{1} u_{2} \ddot{u}_{3}=\frac{1}{3}\left(U_{1} U_{2} V_{3}+U_{1} U_{3} V_{2}+U_{2} U_{3} V_{1}^{\prime}\right)\left(\begin{array}{r}
1 \\
-1
\end{array}\right)
\end{aligned}
$$

All other $F_{n m}$ vanish.

\subsection{The quadratic Taylor coefficients}

Applying the method of Section 1 to (2.14) yields reduced bifurcation equations of the form of (1.10) with (1.11). The functions $A_{i j k}, B_{i j k}$ collected in (1.18) become here

$$
\begin{aligned}
& A_{110}=\theta D \mu_{1}\left(\begin{array}{r}
1 \\
-1
\end{array}\right) \sin k \xi, \quad B_{010}=\theta D \mu_{2}\left(\begin{array}{r}
1 \\
-1
\end{array}\right) \sin (k+1) \xi \\
& A_{200}=D\left(\theta \mu_{1}\right)^{3 / 2} \mu_{2}^{-1 / 2}\left(1+D \mu_{1}\right)\left(1-D \mu_{2}\right)\left(\begin{array}{r}
1 \\
-1
\end{array}\right) \sin ^{2} k \xi \\
& A_{001}=D\left(\theta \mu_{2}\right)^{3 / 2} \mu_{1}^{-1 / 2}\left(1+D \mu_{2}\right)\left(1-D \mu_{1}\right)\left(\begin{array}{r}
1 \\
-1
\end{array}\right) \sin ^{2}(k+1) \xi \\
& B_{100}=2 D \theta^{3 / 2}\left(\mu_{1} \mu_{2}\right)^{1 / 2}\left(1-D^{2} \mu_{1} \mu_{2}\right)\left(\begin{array}{r}
1 \\
-1
\end{array}\right) \sin k \xi \sin (k+1) \xi
\end{aligned}
$$

From (2.16) we obtain the quadratic (in $(x, y, \lambda)$ ) Taylor coefficients of $a$ and $b$,

$$
\begin{aligned}
& a_{110}=f_{1} \theta D \mu_{1} \pi / 2, \quad b_{010}=f_{2} \theta D \mu_{2} \pi / 2 \\
& a_{200}=f_{1} D\left(\theta \mu_{1}\right)^{3 / 2} \mu_{2}^{-1 / 2}\left(1+D \mu_{1}\right)\left(1-D \mu_{2}\right)(4 / 3 k) \\
& a_{001}=f_{1} D\left(\theta \mu_{2}\right)^{3 / 2} \mu_{1}^{-1 / 2}\left(1+D \mu_{2}\right)\left(1-D \mu_{1}\right) P \\
& b_{100}=2 f_{2} D \theta^{3 / 2}\left(\mu_{1} \mu_{2}\right)^{1 / 2}\left(1-D^{2} \mu_{1} \mu_{2}\right) P
\end{aligned}
$$

where

$$
P=4 \mu_{2} / k\left(4 \mu_{2}-\mu_{1}\right) \text {. }
$$

The coefficients (2.17) differ slightly from those of [11] because we have used a different normalization for the eigenfunctions. This does not, however, affect further discussion.

Schaeffer and Golubitsky [11] have shown that $G=(a, y b)$ is contact equivalent (in the sense of singularity theory) to a certain codimension-two normal form (see Section 3) if the following non-degeneracy conditions hold,

$$
a_{200} \neq 0, \quad a_{001} \neq 0, \quad b_{100} \neq 0
$$


and

$$
a_{110} b_{100} \neq a_{200} b_{010} \text {. }
$$

Our purpose is to establish normal forms for $G$ if one of the three inequalities (2.19) fail. For these cases higher order coefficients must be computed which will be pursued in Sections 4-6.

\subsection{Remarks about the perturbed problem}

The ultimate task is to analyse the bifurcations of (2.1) near a double eigenvalue subject to various perturbations. The unperturbed problem (2.14) has been defined such that the double eigenvalue occurs at $\lambda=0\left(B=B_{k}\right)$ because there $A$ is fixed by the condition $A=A_{k}$. In Sections 4-6 we shall also fix $D$ in order to violate one of the three inequalities (2.19). The perturbations are then accounted for in a universal unfolding of the codimension-three normal form to which the reduced bifurcation equation $G$ turns out to be contact equivalent. We do not perform a Liapunov-Schmidt reduction for the perturbed codimension-three cases because this follows the same lines as in the codimension-two case of [11].

It is fairly obvious which perturbations are relevant. At first we can change $D$ and $A$ from their fixed values to perturbed values. Changing only $D$ leads us to the codimension-two cases of [11], while changing $A$ from $A_{k}$ splits the double eigenvalue into two separate (different values of $\lambda$ ) simple eigenvalues. Variations of $\theta$ alone do not induce qualitative changes in the bifurcation diagrams because $\theta$ is only restricted by the condition to be in a certain open interval of the positive real axis.

The second perturbation-suggested in [11]-is not contained in the model equations (2.1). In the underlying chemical reaction, $A$ is a concentration fixed by the experimenter. In practice, however, $A$ will be depleted relative to its diffusity. Taking this effect into account disturbs the trivial solution $u=0$, similar to bifurcations from a simple eigenvalue discussed in [5]. For details we refer to [11].

\subsection{The pseudo-inverse $L_{0}^{-1} Q$}

If $A=A_{k}, B=B_{k}$, then the eigenvalues $\kappa_{|k|-}$ and $\kappa_{|k+1|-}$ of $L$ are zero, with eigenfunctions and co-eigenfunctions given by (2.9) and (2.10), respectively. The other two eigenvalues corresponding to $l=|k|,|k+1|$ in (2.5) are, respectively,

$$
\kappa_{|k|+} \equiv \kappa_{1}=-2 / \pi f_{1}, \quad \kappa_{|k+1|+} \equiv \kappa_{2}=-2 / \pi f_{2},
$$


with eigenfunctions

$$
\psi_{1}=\left(\begin{array}{c}
-D \mu_{2} \\
1+D \mu_{2}
\end{array}\right) \sin k \xi, \quad \psi_{2}=\left(\begin{array}{c}
-D \mu_{1} \\
1+D \mu_{1}
\end{array}\right) \sin (k+1) \xi
$$

and co-eigenfunctions

$$
\psi_{1}^{*}=f_{1}\left(\begin{array}{c}
1+D \mu_{1} \\
\theta D \mu_{1}
\end{array}\right) \sin k \xi, \quad \psi^{*}=f_{2}\left(\begin{array}{c}
1+D \mu_{2} \\
\theta D \mu_{2}
\end{array}\right) \sin (k+1) \xi
$$

In principle it is possible to compute the generalized Green-matrix for $L_{0}^{-1} Q$ explicitly, however, we prefer to work with a sum-representation. Let $u \in X$ be given as a trigonometric series,

$$
u=\frac{2}{\pi} \sum_{j \geqslant 1} u_{j} \sin j \xi, \quad u_{j} \in \mathbb{R}^{2}
$$

Then $L_{0}^{-1} Q$ has the representation

$$
\left(L_{0}^{-1} Q\right) u=\frac{2}{\pi} \sum_{j \geqslant 1}\left(M_{j}^{-1} u_{j}\right) \sin j \xi+\sum_{i=1}^{2} \kappa_{i}^{-1}\left(u, \psi_{i}^{*}\right) \psi_{i}
$$

where the prime at the first sum in (2.25) indicates that the terms $j=|k|$ and $j=|k+1|$ have to be omitted.

In order that stable steady states near the double eigenvalue exist, the real parts of all non-zero eigenvalues of $L$ must be negative. It is easily seen that the eigenvalues $\kappa_{j \pm}$ with $j \neq|k|,|k+1|$ are negative for $A=A_{k}, B=B_{k}$. The eigenvalues (2.21) are negative only if $f_{1}$ and $f_{2}$ are positive, so we must assume that

$$
\theta>\bar{\theta}=\max \left\{\theta_{0}, 1 / \theta_{0}\right\}
$$

where

$$
\theta_{0}=\mu_{2}\left(1+D \mu_{1}\right) / \mu_{1}\left(1+D \mu_{2}\right),
$$

that is, $\bar{\theta}=\theta_{0}$ if $k>0$ and $\bar{\theta}=1 / \theta_{0}$ if $k<0$.

\section{The normal forms}

\subsection{Imperfect bifurcation theory}

Consider a germ of a $C^{\infty}$-mapping $G:\left(\mathbb{R}^{3}, 0\right) \rightarrow\left(\mathbb{R}^{2}, 0\right)$, with variables $(x, y, \lambda) \in \mathbb{R}^{3}$, which is equivariant under the $Z(2)$-action $(x, y, \lambda) \rightarrow(x,-y, \lambda)$, that is,

$$
G(x, y, \lambda)=\left(\begin{array}{c}
a(x, \lambda, z) \\
y b(x, \lambda, z)
\end{array}\right), \quad z=y^{2}
$$


A germ of the form (3.1) satisfying

$$
a(0)=a_{x}(0)=b(0)=0
$$

will be called a bifurcation problem. (If $a_{x}(0) \neq 0$ and $b(0) \neq 0,(3.1)$ reduces to problems discussed in [5] and [7], respectively.) The solution set of the equation $G=0$ is called the bifurcation diagram associated wiht $G$. The question, when are two bifurcation problems qualitatively similar, is made precise via the notion of contact equivalence.

Definition 1. Let $G$ and $H$ be germs of the form (3.1). We say that $G$ and $H$ are contact equivalent if there exists a diffeomorphism

$$
\Phi(x, y, \lambda)=\left(\rho_{1}(x, z, \lambda), y \rho_{2}(x, z, \lambda), \lambda \Lambda(\lambda)\right)
$$

satisfying $\Lambda(0)>0$, and a $Z(2)$-equivariant matrix

$$
T(x, y, \lambda)=\left(\begin{array}{cc}
T_{11}(x, z, \lambda) & y T_{12}(x, z, \lambda) \\
y T_{21}(x, z, \lambda) & T_{22}(x, z, \lambda)
\end{array}\right),
$$

satisfying $\rho_{1 x}(0) T_{11}(0)>0, \rho_{2}(0) T_{22}(0)>0$, such that

$$
H=T G \circ \Phi \text {. }
$$

(Our definition of contact equivalence differs slightly from the one used in [1, $4,6]$ in order to preserve stabilities.) Note that contact equivalence preserves the foliation $\mathbb{R}^{3}=\mathbb{R}^{2} \times \mathbb{R}$, so that the special role of $\lambda$ as a distinguished bifurcation parameter is respected.

Let $G_{\alpha}$ be a $Z(2)$-equivariant perturbation of $G$ depending smoothly on $n$ real unfolding parameters $\alpha=\left(\alpha_{1}, \ldots, \alpha_{n}\right)$ such that $G_{\alpha=0}=0$. We call $G_{\alpha}$ an $n$-parameter unfolding of $G$.

Definition 2. The unfolding $G_{\alpha}$ is versal if, for any other unfolding $G_{\beta}$, $\beta=\left(\beta_{1}, \ldots, \beta_{m}\right)$, there exists a smooth map $\alpha=\psi(\beta)$ so that $G_{\psi(\beta)}$ is contact equivalent to $G_{\beta}$. The unfolding $G_{\alpha}$ is universal if it is versal and possesses the minimum number of unfolding parameters needed for being versal. This minimum number is called the codimension, $\operatorname{cod} G$, of $G$.

Roughly, the codimension is a measure of the degeneracy $G$ possesses at the origin. If $G_{\alpha}$ is a universal unfolding of a bifurcation problem $G$, we call the solution set of the equation $G_{\alpha}=0$ the perturbed bifurcation diagram associated with $G$.

By using the general results of [6], a classification of bifurcation problems up to codimension four has been carried out in [4], in terms of a list of normal forms. To any normal form $G_{0}$ belongs a set of expressions in terms of the $a_{i j k}, b_{i j k}$, which must be zero if an arbitrary bifurcation problem $G$ be contact equivalent to $G_{0}$. They are called degeneracy conditions for the normal form. The number of degeneracy conditions equals $\operatorname{cod} G_{0}$. Since we have specified bifurcation problems to satisfy (3.2), one of the degeneracy conditions is always $a_{010}=0$, that is, 
the codimension of any bifurcation problem is $\geqslant 1$. There is, furthermore, another set of expressions in terms of the $a_{i j k}, b_{i j k}$ which must be non-zero. These are called non-degeneracy conditions. An arbitrary bifurcation problem $G$ is contact equivalent to the normal form $G_{0}$ if and only if the degeneracy and non-degeneracy conditions are satisfied.

In what follows we collect those normal forms up to codimension three which are relevant for the Brusselator, together with the corresponding degeneracy and non-degeneracy conditions. The conditions are specified here to bifurcation problems satisfying also the first equation of (1.11) (for the general case see [4]), that is,

$$
a_{0 n 0}=0, \quad n \in N
$$

\subsection{The codimension-two normal form}

First we state a normal form which is contact equivalent to the one discussed in [11]. It has the form

$$
\left(\begin{array}{l}
\varepsilon_{1}\left(x^{2}-\lambda^{2}+\varepsilon z\right) \\
\varepsilon_{2} y(x-\rho \lambda)
\end{array}\right), \quad \rho \neq 1, \rho \geqslant 0
$$

with the $\varepsilon$ 's being \pm 1 . The Schaeffer-Golubitsky normal form is given by

$$
\left(\begin{array}{l}
\varepsilon_{1}^{\prime}\left(x^{2}+\varepsilon^{\prime} x \lambda+\varepsilon z\right) \\
\varepsilon_{2}^{\prime} y\left(\rho^{\prime} x+\lambda\right)
\end{array}\right), \quad \rho^{\prime} \neq 0, \varepsilon^{\prime} .
$$

Assuming $\varepsilon^{\prime}-2 / \rho^{\prime} \neq 0$ and choosing

$$
\Phi=\left(\varepsilon^{\prime \prime} x-\varepsilon^{\prime} \lambda, y, 2 \lambda\right), T_{11}=\varepsilon^{\prime \prime}, T_{22}=1 /\left|\rho^{\prime}\right|, T_{12}=T_{21}=0,
$$

where $\varepsilon^{\prime \prime}=\operatorname{sgn}\left(\varepsilon^{\prime}-2 / \rho^{\prime}\right)$, we find that (3.5) is contact equivalent to (3.4) with

$$
\varepsilon_{1}=\varepsilon_{1}^{\prime} \varepsilon^{\prime \prime}, \varepsilon_{2}=\varepsilon^{\prime \prime} \varepsilon_{2}^{\prime} \operatorname{sgn} \rho^{\prime}, \rho=\left|\varepsilon^{\prime}-2 / \rho^{\prime}\right| .
$$

Observe that the bifurcation problem $G$ occuring in the Brusselator satisfies (3.3). This implies that the equation $G=0$ possesses the solution $x=y=0$ corresponding to the trivial solution $u=0$ of (2.14). If $G$ is transformed to the normal form (3.5), then the trivial solution also corresponds to the branch $x=y=0$ of the bifurcation diagram associated with (3.5). This branch transforms onto $(x, y)=\left(\varepsilon^{\prime} \varepsilon^{\prime \prime} \lambda, 0\right)$ under the contact equivalence from (3.5) to (3.4). A similar situation occurs with the normal forms of Subsections 3.3-5. (We could choose alternative normal forms satisfying (3.3), but for discussing the bifurcation diagrams it is more convenient to use the normal forms introduced here. Note that contact equivalence does not distinguish between the trivial solution $x=y$ $=0$ and any solution $(x, y)=(x(\lambda), 0)$ depending smoothly on $\lambda$.) 


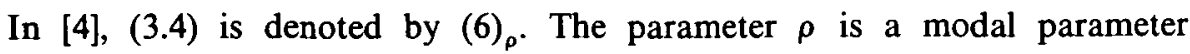
parametrizing the largest family of perturbations of (3.4) siuch that no two perturbations in this family are contact equivalent under the smooth equivalence of Definition 1. The codimension of (3.4) is $3^{*}$ where the asterisk indicates the presence of the modal parameter. We regard $(6)_{\rho}$ as a codimension-two problem because its topological codimension (contact codimension minus number of modal parameters) is two.

The degeneracy conditions $\left(a_{010}=0\right)$ for type $(6)_{\rho}$ are always satisfied in virtue of (3.3). The non-degeneracy conditions are $a_{110} \neq 0, b_{010} \neq 0$ (satisfied for the Brusselator) and the inequalities (2.19), (2.20). If they are satisfied, the modal parameter and the signs in (3.4) are given by

$$
\begin{gathered}
\varepsilon=\operatorname{sgn}\left(a_{001} a_{200}\right), \\
\varepsilon_{1}=\operatorname{sgn}\left(\mu a_{200}\right), \varepsilon_{2}=\operatorname{sgn}\left(\mu b_{100}\right), \rho=|\mu| \text { if } \mu \neq 0, \\
\varepsilon_{1}=\operatorname{sgn} a_{200}, \varepsilon_{2}=\operatorname{sgn} b_{100}, \rho=0 \text { if } \mu=0,
\end{gathered}
$$

where

$$
\mu=1-2 a_{200} b_{010} / a_{110} b_{100}
$$

\subsection{Type (9) 31}

Normal form:

$$
\left(\begin{array}{l}
\varepsilon_{1}\left(x^{3}-\varepsilon^{\prime} x \lambda+\varepsilon z\right) \\
\varepsilon_{2} y(x-\lambda)
\end{array}\right)
$$

Degeneracy condition:

$$
a_{200}=0
$$

Non-degeneracy condition:

$$
a_{300} a_{110} a_{001} b_{100} b_{010} \neq 0
$$

The signs in (3.7) are given by

$$
\begin{aligned}
& \varepsilon_{1}=\operatorname{sgn} a_{300}, \quad \varepsilon_{2}=-\operatorname{sgn} b_{010}, \\
& \varepsilon^{\prime}=-\operatorname{sgn}\left(a_{300} a_{110}\right), \quad \varepsilon=\varepsilon_{1} \varepsilon_{2} \operatorname{sgn}\left(b_{100} a_{001}\right)
\end{aligned}
$$

\subsection{Type (8) 221}

Normal form:

$$
\left(\begin{array}{l}
\varepsilon_{1}\left(x^{2}-\lambda^{2}+\varepsilon z\right) \\
\varepsilon_{2} y\left(x^{2}-\varepsilon^{\prime} \lambda\right)
\end{array}\right)
$$


Degeneracy condition:

$$
b_{100}=0 \text {. }
$$

Non-degeneracy condition:

$$
a_{200} a_{110} a_{001} b_{010} c_{8} \neq 0,
$$

where

$$
c_{8}=b_{200} a_{001}-a_{200} b_{001} .
$$

The signs in (3.11) are given by

$$
\begin{aligned}
& \varepsilon_{1}=\operatorname{sgn} a_{200}, \quad \varepsilon_{2}=\operatorname{sgn}\left(a_{001} c_{8}\right) \\
& \varepsilon=\operatorname{sgn}\left(a_{001} a_{200}\right), \quad \varepsilon^{\prime}=-\varepsilon_{2} \operatorname{sgn} b_{010} .
\end{aligned}
$$

\subsection{Type $(6 a)_{\rho, \kappa}$}

Normal form:

$$
\left(\begin{array}{cl}
\varepsilon_{1}\left(x^{2}-\lambda^{2}+\varepsilon z^{2}+2 \kappa z \lambda\right) \\
\varepsilon_{2} y(x-\rho \lambda)
\end{array}\right), \quad \begin{aligned}
& \rho \neq 1, \rho \geqslant 0 \\
& \varepsilon\left(\rho^{2}-1\right) \neq \kappa^{2}
\end{aligned}
$$

Degeneracy condition:

$$
a_{001}=0
$$

Non-degeneracy condition:

$$
a_{200} b_{100} p r d \neq 0
$$

where

$$
\begin{aligned}
& d=4 r p-q^{2} \\
& p=a_{200} b_{001}^{2}-a_{101} b_{001} b_{100}+a_{002} b_{100}^{2} \\
& q=a_{011} b_{100}^{2}-2 a_{110} b_{100} b_{001}+2 a_{200} b_{001} b_{010}-a_{101} b_{010} b_{100} \\
& r=b_{010}\left(a_{200} b_{010}-a_{110} b_{100}\right) .
\end{aligned}
$$

Type (6a) $)_{\rho, \kappa}$ has codimension $5^{* *}$, that is, there are two modal parameters $\rho, \kappa$ which have to satisfy the inequalities in (3.16). These separate $(\rho, \kappa)$-space into various open regions which are distinguished as follows.

$$
\begin{aligned}
& \varepsilon=+1: C I\left(\rho^{2}<1\right), C I I\left(1<\rho^{2}<\kappa^{2}+1\right), C I I I\left(\rho^{2}>\kappa^{2}+1\right) \\
& \varepsilon=-1: D I\left(\rho^{2}+\kappa^{2}<1\right), D I I\left(1-\kappa^{2}<\rho^{2}<1\right), D I I I\left(\rho^{2}>1\right) .
\end{aligned}
$$


The different modal cases of (3.20) are determined by

$$
\begin{aligned}
& C I:( \pm, \pm, \mp,-), C I I:( \pm, \pm, \pm,-), C I I I:( \pm, \pm, \pm,+), \\
& D I:( \pm, \pm, \mp,+), D I I:( \pm, \mp, \mp,-), D I I I:( \pm, \mp, \pm,-),
\end{aligned}
$$

where in the brackets in (3.21) the signs

$$
\left(\operatorname{sgn} a_{200}, \operatorname{sgn} p, \operatorname{sgn} r, \operatorname{sgn} d\right)
$$

are collected. The regions defined by (3.20) are not connected in the $(\rho \geqslant 0, \kappa)$-half plane. We have to specify (3.20) further by

$$
\begin{gathered}
\operatorname{sgn} \kappa=\operatorname{sgn}\left(q a_{200}\right) \text { if } q \neq 0, \\
\kappa=0 \text { if } q=0 .
\end{gathered}
$$

The signs $\varepsilon_{1}, \varepsilon_{2}$ and $\rho$ are given by $(3.6 \mathrm{~b}, \mathrm{c})$.

\subsection{Type (7)}

It will be shown in Sections $4-6$ that the types $(9)_{31},(8)_{221},(6 a)_{\rho, \kappa}$ occur in the Brusselator if one of the non-degeneracy conditions (2.19) for type (6) $)_{\rho}$ is not satisfied. For completeness we present also the normal form $(7)_{2}$ corresponding to the non-degeneracy condition $a_{110} b_{100}=a_{200} b_{010}$ which violates (2.20). It has the form

$$
\left(\begin{array}{c}
\varepsilon_{1}\left(x^{2}-\lambda^{2}+\varepsilon z\right) \\
\varepsilon_{2} y\left(x-\varepsilon^{\prime} \lambda-\varepsilon^{\prime \prime} \lambda^{2}\right)
\end{array}\right) .
$$

Since this case will not be discussed further, non-degeneracy conditions etc. are omitted (see [4]). For perturbed bifurcation diagrams associated with $(7)_{2}$ we refer to [1].

\section{Liapunov-Schmidt reduction for type $(9)_{31}$}

From (2.17) and (3.8) we infer that the degeneracy conditions for type (9) ${ }_{31}$ are satisfied if

$$
D \mu_{2}=1
$$

so that

$$
A_{k}=\left(\theta \mu_{1} / \mu_{2}\right)^{1 / 2}, \quad B_{k}=2\left(1+\mu_{1} / \mu_{2}\right), \quad \theta_{0}=\left(\mu_{1}+\mu_{2}\right) / 2 \mu_{1} .
$$


The non-degeneracy condition (3.9) implies that we have to compute the coefficient $a_{300}$ in addition to those given in (2.17). Noting that (4.1) implies $A_{200}=0$, hence $V_{200}=0$, the first equation in (1.19) yields $A_{300}=F_{30} \phi_{1}^{3}$, that is,

$$
A_{300}=-\theta^{2}\left(\mu_{1} / \mu_{2}\right)^{2}\left(1+\mu_{1} / \mu_{2}\right)\left(\begin{array}{c}
1 \\
-1
\end{array}\right) \sin ^{3} k \xi \text {. }
$$

From (4.3) $a_{300}$ is readily computed,

$$
a_{300}=-f_{1} \mathbf{\nu}^{2}\left(\ddot{\mu}_{1} / \mu_{2}\right)^{2}\left(1+\mu_{1} / \mu_{2}\right) 3 \pi / \text { ió. }
$$

The remaining coefficients entering (3.9) are

$$
\begin{aligned}
& a_{110}=f_{1} \theta \frac{\mu_{1}}{\mu_{2}} \frac{\pi}{2}, b_{010}=f_{2} \theta \frac{\pi}{2} \\
& a_{001}=2 f_{1} \theta^{3 / 2}\left(\mu_{2} / \mu_{1}\right)^{1 / 2}\left(1-\mu_{1} / \mu_{2}\right) P \\
& b_{100}=2 f_{2} \theta^{3 / 2}\left(\mu_{1} / \mu_{2}\right)^{1 / 2}\left(1-\mu_{1} / \mu_{2}\right) P .
\end{aligned}
$$

Noting that $\operatorname{sgn} a_{001}=\operatorname{sgn} b_{100}=\operatorname{sgn} k$, the signs in (9) $)_{31}$ are obvious.

THEOREM 1. Let $\theta>\bar{\theta}$ and $D \mu_{2}=1$. Then the bifurcation problem resulting from the unperturbed problem (2.14) via Liapunov-Schmidt reduction is contact equivalent to the normal form $(9)_{31}, E q .(3.7)$, with signs

$$
\varepsilon_{1}=\varepsilon_{2}=-1, \quad \varepsilon=\varepsilon^{\prime}=1 \text {. }
$$

\section{Liapunov-Schmidt reduction for type $(8)_{221}$}

The degeneracy conditions for type $(8)_{221}\left(b_{100}=0\right)$ are satisfied if

$$
D^{2} \mu_{1} \mu_{2}=1
$$

hence,

$$
A_{k}=\sqrt{\theta}, B_{k}=\frac{(2 k+1)^{2}}{k(k+1)}, \theta_{0}=\frac{k+1}{k} .
$$

In order to find the signs in (8) 221 we need the Taylor coefficients $b_{200}$ and $b_{001}$.

\subsection{Computation of $b_{200}$}

Observe that, in virtue of (5.1), $B_{100}=0$, hence $U_{100}=0$ so that, from (1.19),

$$
B_{200}=2 F_{20} \phi_{2} V_{200}+3 F_{30} \phi_{1}^{2} \phi_{2} \text {. }
$$

The second term in (5.3), $B_{200}^{3} \equiv 3 F_{30} \phi_{1}^{2} \phi_{2}$, is readily found to be

$$
B_{200}^{3}=-\left(\theta^{2} / 3 \mu_{2}\right)(2 k+1)(3 k+2)\left(\begin{array}{c}
1 \\
-1
\end{array}\right) \sin ^{2} k \xi \sin (k+1) \xi
$$


The first term in (5.3) requires to compute $V_{200}=-L_{0}^{-1} Q A_{200}$, where

$$
A_{200}=v_{2}\left(\begin{array}{c}
1 \\
-1
\end{array}\right) \sin ^{2} k \xi
$$

with

$$
v_{2}=-\theta^{3 / 2} k(2 k+1) /(k+1)^{3} .
$$

To achieve this we write $A_{200}$ as a Fourier series,

$$
A_{200}=\frac{2}{\pi} v_{2} \sum_{j \geq 1} P_{j k} \sin j \xi
$$

where

$$
P_{j l}=\int_{0}^{\pi} d \xi \sin ^{2} l \xi \sin j \xi
$$

Note that $P_{k, k+1}=P$ (cf. (2.18)) and $P_{J l}=0$ if $j$ is even. Applying the representation (2.25) to (5.7) gives $V_{200}=V_{200}^{1}+V_{200}^{2}$, with

$$
\begin{aligned}
& V_{200}^{1}=-\frac{2}{\pi} v_{2} \sum_{j \neq|k|}\left(P_{j k} / m_{j}\right)\left(\begin{array}{c}
-\theta D j^{2} \\
1+D j^{2}
\end{array}\right) \sin j \xi, \\
& V_{200}^{2}=-\frac{8 \theta^{3 / 2} D(2 k+1-k \theta)}{3 \pi(2 k+1)\left(\theta-\theta_{0}\right)^{2}}\left(\begin{array}{c}
-1 \\
1+D \mu_{1}
\end{array}\right) \sin k \xi .
\end{aligned}
$$

In (5.8a) we have used the notation

$$
m_{j}=\operatorname{det} M_{j}=\theta D^{2}\left(\mu_{1}-j^{2}\right)\left(\mu_{2}-j^{2}\right) .
$$

Setting $B_{200}^{i}=2 F_{20} \phi_{2} V_{200}^{i}(i=1,2)$ we obtain from (5.8)

$$
\begin{aligned}
& B_{200}^{1}=\frac{4}{\pi} \theta^{2}(2 k+1)\left(\begin{array}{c}
1 \\
-1
\end{array}\right) \sum_{j \neq|k|} \frac{P_{j k}}{\mu_{2}-j^{2}} \sin j \xi \sin (k+1) \xi, \\
& B_{200}^{2}=\frac{16 \theta^{2} D\left(\theta-\theta_{1}\right)}{3 \pi \mu_{1}\left(\theta-\theta_{0}\right)}\left(\begin{array}{c}
1 \\
-1
\end{array}\right) \sin k \xi \sin (k+1) \xi,
\end{aligned}
$$

where

$$
\theta_{1}=(2 k+1) / k \text {. }
$$

Using (5.3) and (5.10) in (1.21) yields

$$
b_{200}=b_{200}^{1}+b_{200}^{2}+b_{200}^{3} \text {, }
$$

where

$$
\begin{aligned}
& b_{200}^{1}=\frac{4}{\pi} f_{2} \theta^{2}(2 k+1) S_{2}, \\
& b_{200}^{2}=\frac{16 \theta^{2} f_{2} P\left(\theta-\theta_{1}\right)}{3 \pi k^{3}(k+1)\left(\theta-\theta_{0}\right)}, \\
& b_{200}^{3}=-\left(f_{2} \theta^{2} \pi / 24 \mu_{2}\right)(3 k+2)(2 k+1) .
\end{aligned}
$$


In (5.13a), $S_{2}$ denotes the sum

$$
S_{2}=\sum_{j \neq|k|} P_{j k} P_{j, k+1} /\left(\mu_{2}-j^{2}\right) .
$$

In order to compute $S_{2}$, consider the generalized Green function $K_{n}(\xi, \eta)$ ( $n$ an integer) corresponding to the differential operator $(\partial / \partial \xi)^{2}+n^{2}$, with homogeneous Dirichlet boundary conditions. The sum representation for $K_{n}$ is

$$
K_{n}(\xi, \eta)=\frac{2}{\pi} \sum_{j \neq|n|} \frac{\sin j \xi \sin j \eta}{n^{2}-j^{2}}
$$

Alternatively, we can write

$$
\begin{gathered}
K_{n}(\xi, \eta)=\frac{\xi}{n \pi} \cos n \xi \sin n \eta+\frac{1}{\mathrm{n} \pi}\left(\eta \cos n \eta-\frac{1}{2 n} \sin n \eta\right) \sin n \xi \\
- \begin{cases}\frac{1}{n} \cos n \eta \sin n \xi, & \xi<\eta \leqslant \pi \\
\frac{1}{n} \sin n \eta \cos n \xi, & 0 \leqslant \eta<\xi .\end{cases}
\end{gathered}
$$

Consider then the function

$$
U_{2}(\xi)=\sin ^{2} k \xi-\frac{8}{3 k \pi} \sin k \xi
$$

which is orthogonal to $\sin k \xi$ with respect to the $L_{2}$-inner product. By using the sum representation (5.15a) one readily verifies that

$$
S_{2}=\frac{\pi}{2} \int_{0}^{\pi} d \xi \int_{0}^{\pi} d \eta K_{k+1}(\xi, \eta) \sin ^{2}(k+1) \xi U_{2}(\eta) .
$$

Substituting (5.15b) into (5.17) allows the computation of an explicit expression for $S_{2}$ which, when inserted into (5.13a) yields

$$
b_{200}^{1}=f_{2} \theta^{2}\left(\frac{(2 k+1) \pi}{2 \mu_{2}}-\frac{16}{3 k \pi} P\right) .
$$

\subsection{Computation of $b_{001}$}

Here one has to compute first $V_{001}=-L_{0}^{-1} Q A_{001}$ with $A_{001}$ given in (2.16). Then $V_{001}$ is substituted into the last equation of (1.19) yielding $B_{001}$ from which $b_{001}$ is obtained via (1.21). Proceeding in the same way as in the last Subsection we find

$$
b_{001}=b_{001}^{1}+b_{001}^{2}+b_{001}^{3}
$$


where

$$
\begin{aligned}
& b_{001}^{1}=-\frac{4}{\pi} f_{2} \theta^{2}\left(\mu_{2} / \mu_{1}\right)^{2}(2 k+1) S_{1} \\
& b_{001}^{2}=-\frac{4 \theta^{2} f_{2} P^{2}(k+1)^{3}\left(\theta-\theta_{1}\right)}{\pi k^{4}\left(\theta-\theta_{0}\right)} \\
& b_{001}^{3}=-\left(3 f_{2} \theta^{2} \pi \mu_{2} / 16 k^{3}\right)(2 k+1) .
\end{aligned}
$$

In (5.20a), $S_{1}$ denotes the sum

$$
\begin{aligned}
S_{1} & =\sum_{j \neq|k|} P_{J, k+1}^{2} /\left(\mu_{2}-j^{2}\right), \\
& =\frac{\pi}{2} \int_{0}^{\pi} d \xi \int_{0}^{\pi} d \eta K_{k+1}(\xi, \eta) \sin ^{2}(k+1) \xi U_{1}(\eta),
\end{aligned}
$$

where

$$
U_{1}(\xi)=\sin ^{2}(k+1) \xi-\frac{2}{\pi} P \sin k \xi
$$

From (5.21) we obtain the final result for $b_{001}^{1}$,

$$
b_{001}^{1}=f_{2} \theta^{2}\left(\mu_{2} / 2 \mu_{1}^{2}\right)\left\{\frac{8}{\pi} \mu_{2} P^{2}-(2 k+1) \pi\right\} .
$$

\subsection{The signs in $(8)_{221}$}

We are now in the position to determine the signs in $(8)_{221}$. The quadratic coefficients entering the non-degeneracy condition (3.13) are

$$
\begin{aligned}
& a_{110}=f_{1} \theta k \pi / 2(k+1), \quad b_{010}=f_{2} \theta(k+1) \pi / 2 k \\
& a_{200}=-4 f_{1} \theta^{3 / 2}(2 k+1) / 3(k+1)^{3} \\
& a_{001}=f_{1} \theta^{3 / 2} P(k+1)(2 k+1) / k^{3} .
\end{aligned}
$$

From (5.23) and the results of Subsection 5.1-2 we obtain for $c_{8}$, Eq. (3.14),

$$
c_{8}=-\pi f_{1} f_{2} \theta^{7 / 2}(2 k+1)^{2}\left(15 k^{3}+40 k^{2}+42 k+12\right) / 12 k^{4}(k+1) .
$$

Observe that $c_{8}$ is negative for $\theta>\bar{\theta}$ and all relevant $k$ ( $k$ odd, $k \geqslant 1$ or $k \leqslant-3$ ), hence the signs in (3.11) are obvious.

THEOREM 2. Let $\theta>\bar{\theta}$ and $D^{2} \mu_{1} \mu_{2}=1$. Then the bifurcation problem resulting from the unperturbed problem (2.14) via Liapunov-Schmidt reduction is contact equivalent to the normal form (8) $221, E q$. (3.11), with signs

$$
\varepsilon_{1}=\varepsilon_{2}=\varepsilon=-1, \quad \varepsilon^{\prime}=1 \text {. }
$$


We note that the signs in (5.25) are consistent with stability considerations: only if $\varepsilon_{1}=\varepsilon_{2}=-1$ the solution $(x, y)=(-\lambda, 0)$ of $G=0$, corresponding to the trivial solution $u=0$ in (2.14), is stable for $\lambda<0$ and unstable for $\lambda>0$.

\section{Liapunov-Schmidt reduction for type $(6 a)_{p, \kappa}$}

The degeneracy conditions $\left(a_{001}=0\right)$ for $(6 a)_{\rho, \kappa}$ are satisfied if

$$
D \mu_{1}=1 \text {, }
$$

so that

$$
A_{k}=\left(\theta \mu_{2} / \mu_{1}\right)^{1 / 2}, \quad B_{k}=2\left(1+\mu_{2} / \mu_{1}\right), \quad \theta_{0}=2 \mu_{2} /\left(\mu_{1}+\mu_{2}\right) .
$$

In order to decide which of the subtypes of $(6 a)_{\rho, \kappa}$ (cf. (3.20), (3.21)) occur in the Brusselator, we have to estimate the signs of $a_{200}$ and $p, q, r, d$, defined in (3.19). The latter depend on the non-vanishing quadratic Taylor-coefficients and on $a_{101}, a_{002}, a_{011}, b_{001}$.

\subsection{Computation of Taylor coefficients}

Since (6.1) implies $A_{001}=0$, hence $V_{001}=0$, it follows that $B_{001}=F_{30} \phi_{2}^{3}$, so

$$
B_{001}=-\left(\theta \mu_{2} / \mu_{1}\right)^{2}\left(1+\mu_{2} / \mu_{1}\right)\left(\begin{array}{c}
1 \\
-1
\end{array}\right) \sin ^{3}(k+1) \xi
$$

and

$$
b_{001}=-3 f_{2} \theta^{2} \pi \mu_{2}^{2}\left(\mu_{1}+\mu_{2}\right) / 16 \mu_{1}^{3} .
$$

The computation of $a_{011}$ is also straightforward. First note that $B_{010}$ (cf. (2.16)) is orthogonal to all eigenvalues of $L^{*}$ except $\psi_{2}^{*}$ (cf. (2.23)). This implies that only the second term in (2.25) contributes to $U_{010}$. Therefore, $U_{010}$ is easily computed and we obtain $a_{011}$ by means of the third equation in (1.19) and (1.21). The result is

$$
a_{011}=f_{1} \theta^{3 / 2}\left(\frac{k+1}{k}\right)^{3} P(1-2 J)
$$

where

$$
J(\theta)=\frac{1-\theta \theta_{0} / 2}{1-\theta \theta_{0}}
$$

For evaluating $a_{002}$ observe that $V_{001}=0$ and $F_{40}=0$. Hence, from (1.20) and (1.21),

$$
a_{002}=\left(2 F_{20} \phi_{2} U_{001}, \phi_{1}^{*}\right)
$$


where $U_{001}=-L_{0}^{-1} Q B_{001}$. Splitting the "sine-cubed" in (6.3) into

$$
\sin ^{3}(k+1) \xi=\frac{3}{4} \sin (k+1) \xi-\frac{1}{4} \sin ^{3}(k+1) \xi
$$

shows that, when applying (2.25) to $B_{001}$, only one term in the first sum survives. Therefore, $U_{001}$ is easily computed and we obtain from (6.7),

$$
a_{002}=\frac{3}{2} f_{1} \theta^{5 / 2}\left(\frac{k+1}{k}\right)^{5}\left(1+\mu_{2} / \mu_{1}\right) P\left\{J-\mu_{1}^{2} /\left(9 \mu_{2}-\mu_{1}\right)\left(16 \mu_{2}-\mu_{1}\right)\right\} \text {. }
$$

The last coefficient, $a_{101}$, is found in the same way as $b_{200}$ in Subsection 5.1. We obtain

$$
a_{101}=a_{101}^{1}+a_{101}^{2}+a_{101}^{3}
$$

where

$$
\begin{aligned}
& a_{101}^{1}=\frac{8}{\pi} f_{1} \theta^{2}\left(\mu_{2} / \mu_{1}\right)(2 k+1) S_{3} \\
& a_{101}^{2}=\frac{8}{\pi} f_{1} \theta^{2}\left(\mu_{2} / \mu_{1}^{2}\right)(2 k+1) P^{2} J \\
& a_{101}^{3}=-\frac{\pi}{4} f_{1} \theta^{2}\left(\mu_{2} / \mu_{1}\right)\left(1+2 \mu_{2} / \mu_{1}\right) .
\end{aligned}
$$

In (6.10a), $S_{3}$ denotes the sum

$$
S_{3}=\sum_{j \neq|k+1|} Q_{j k}^{2} /\left(\mu_{1}-j^{2}\right)
$$

where

$$
Q_{J k}=\int_{0}^{\pi} d \xi \sin j \xi \sin k \xi \sin (k+1) \xi,
$$

so that $Q_{j k}=0$ if $j$ is odd. Defining

$$
U_{3}(\xi)=\sin k \xi \sin (k+1) \xi-\frac{2}{\pi} P \sin (k+1) \xi,
$$

$S_{3}$ can be evaluated by substituting (5.19b) into

$$
S_{3}=\frac{\pi}{2} \int_{0}^{\pi} d \xi \int_{0}^{\pi} d \eta K_{k}(\xi, \eta) \sin k \xi \sin (k+1) \xi U_{3}(\xi) .
$$

From (6.13) and (6.10a) we obtain the final expression for $a_{101}^{1}$,

$$
\begin{aligned}
& a_{101}^{1}=f_{1} \theta^{2}\left(\mu_{2} / \mu_{1}\right)\left\{\frac{(2 k+1) \pi}{4 \mu_{1}-\mu_{2}}+\frac{8}{\pi} P\right\} \quad \text { if } k \neq 1, \\
& a_{101}^{1}=24 f_{1} \theta^{2}\left(\frac{32}{45 \pi}-\frac{5 \pi}{32}\right) \text { if } k=1 .
\end{aligned}
$$


For completeness we present also the quadratic coefficients. If (6.1) holds they reduce to

$$
\begin{array}{ll}
a_{200}=-8 f_{1} \theta^{3 / 2}(2 k+1) / 3 \mu_{1}(k+1), & a_{110}=f_{1} \theta \frac{\pi}{2} \\
b_{100}=-2 f_{2} \theta^{3 / 2}(2 k+1)(k+1) P / k^{3}, & b_{010}=f_{2} \theta\left(\mu_{2} / \mu_{1}\right) \frac{\pi}{2} .
\end{array}
$$

\subsection{Estimating the signs of $p, q, r, d$}

Using the results of the last Subsection, we are now in the position to estimate the signs of $p, q, r, d$ defined in (3.19). These terms can be written in the form

$$
\begin{aligned}
& p=f_{1} f_{2}^{2} \theta^{11 / 2}\left(p_{1}+p_{2} J\right) \\
& q=f_{1} f_{2}^{2} \theta^{9 / 2} q_{1}, \quad r=f_{1} f_{2}^{2} \theta^{7 / 2} r_{1} \\
& d=f_{1}^{2} f_{2}^{4} \theta^{9}\left(d_{1}+d_{2} J\right)
\end{aligned}
$$

where

$$
d_{1}=4 p_{1} r_{1}-q_{1}^{2}, \quad d_{2}=4 p_{2} r_{1}
$$

The $p_{t}, q_{1}$ etc. are rational functions of $k$, explicit expressions are given in Appendix A.

The following result is proved in Appendix B.

Proposition 1. If $\theta>\bar{\theta}$, then $\operatorname{sgn} r=-\operatorname{sgn} k$ and $q<0$.

Next we estimate $p$ and $d$.

Proposition 2. Let $\theta>\overline{\boldsymbol{\theta}}$.

(i) $p>0, d<0$ if $k \geqslant 1$.

(ii) $p>0$ if $k \leqslant-5$.

(iii) If $k \leqslant-5$, then $d$ has a unique zero $\theta_{d}>\bar{\theta}$ such that $d<(>) 0$ for $\theta>(<) \theta_{d}$.

(iv) If $k=-3$, then $p$ and $d$ have zeros $\theta_{p}$ and $\theta_{d}$, respectively, satisfying $\bar{\theta}<\theta_{d}<\theta_{p}$. Moreover, $p>(<) 0$ if $\theta<(>) \theta_{p}$ and $d>(<) 0$ if $\theta<(>) \theta_{d}$. 
Proof. The functions $p_{1}+J(\theta) p_{2}$ and $d_{1}+J(\theta) d_{2}$ have unique zeros $\theta_{p}$ and $\theta_{d}$, respectively, given by

$$
\theta_{p}=\frac{p_{1}+p_{2}}{\theta_{0}\left(p_{1}+p_{2} / 2\right)}, \quad \theta_{d}=\frac{d_{1}+d_{2}}{\theta_{0}\left(d_{1} 2+d_{2} / 2\right)} .
$$

An analogous estimation to that of Appendix B shows that $\theta_{p}, \theta_{d}<\theta_{0}$ if $k \geqslant 1$, $\theta_{p}<\theta_{0}^{-1}$ if $k \leqslant-5$ and $\theta_{d}>\theta_{0}^{-1}$ if $k \leqslant-3$. Similarly we find that $p_{1}+p_{2} / 2$ $>0$ for $k \neq-3$ and $d_{1}+d_{2} / 2<0$ for all odd $k$. Hence, $p_{1}+J p_{2}$ and $d_{1}+J d_{2}$ approach for $k \neq-3$ a positive value and for all odd $k$ a negative value, respectively, when $\theta$ goes to infinity. This proves (i)-(iii). If $k=-3$, a direct computation yields

$$
\bar{\theta}=1.63, \quad \theta_{p}=3.59, \quad \theta_{d}=1.93
$$

and $p_{1}+p_{2} / 2=-0.015$, which proves (iv).

It is worth noting that both $\theta_{p}$ and $\theta_{d}$ approach $1 / \theta_{0}$ (the pole of $J$ ) for large $k$,

$$
\begin{aligned}
& \theta_{p}-1 / \theta_{0}=\left(1024 / 27 \pi^{2}\right) k^{-3}+O\left(k^{-4}\right) \\
& \theta_{d}-1 / \theta_{0}=\left(2048 / 81 \pi^{2}\right) k^{-4}+O\left(k^{-5}\right),
\end{aligned}
$$

thus $\theta_{d}$ approaches $1 / \theta_{0}$ rapidly. In fact, the numerical values for $k=-5$ are $1 / \theta_{0}=1.28$ and $\theta_{d}=1.29$ so that the region where $d>0$ is very small.

Recalling that $a_{200}<0$ and $\operatorname{sgn} \kappa=\operatorname{sgn}\left(q a_{200}\right)=1$, the modal cases of $(6 a)_{\rho, \kappa}$ are now obvious by virtue of Proposition 2 and (3.21).

THEOREM 3. Assume that $\theta>\bar{\theta}, \theta \neq \theta_{p}, \theta_{d}, D \mu_{1}=1$ and let $G=(a, y b)$ be the bifurucation problem resulting from the unperturbed problem (2.14) via Liapunov$S c h m i d t$ reduction. Then $G$ is contact equivalent to a member of the family $(6 \mathrm{a})_{\rho, \kappa}$ with $\kappa>0, \varepsilon_{1}=\varepsilon_{2}=(-) 1$ if $k \leqslant-3(k \geqslant 1)$ and modal cases as follows.

$$
\begin{array}{ll}
k \geqslant 1: D I I & \\
k=-3: D I\left(\bar{\theta}<\theta<\theta_{d}\right), & D I I\left(\theta_{d}<\theta<\theta_{p}\right), C I\left(\theta>\theta_{p}\right) \\
k \leqslant-5: D I\left(\bar{\theta}<\theta<\theta_{d}\right), & D I I\left(\theta>\theta_{d}\right) .
\end{array}
$$

Note that the trivial solution $u=0$ of (2.14) corresponds to the branches $(x, y)=(-\lambda, 0)$ and $(x, y)=(\lambda, 0)$ of the bifurcation diagram associated with $(6 \mathrm{a})_{\rho, x}$ if $k \geqslant 1$ and $k \leqslant-3$, respectively.

Observe that for $k \leqslant-3$ and $\theta=\theta_{d}$ the nondegeneracy conditions for (6a) $)_{\rho, \kappa}$ are violated. In this case the degeneracy conditions for a type called (6c) in [4] which has codimension $5^{*}$ are fulfilled. Similarly, if $k=-3$ and $\theta=\theta_{p}$, the degeneracy conditions for another degenerate bifurcation of codimension $5^{*}$ 
called (6b) $)_{\rho}$ in [4] are satisfied. On the other hand all nondegeneracy conditions are satisfied in the cases of (8) 221 and (9) $)_{31}$ if $\theta>\bar{\theta}$. Consequently, the only codimension-4 problems which can appear in the Brusselator are the types $(6 \mathrm{~b}){ }_{\rho}$ and $(6 \mathrm{c})_{\rho}$.

\section{Universal unfoldings and subordinate bifurcations}

\subsection{Universal unfoldings}

The universal unfolding of a codimension-3 normal form $G(x, y, \lambda)$ introduced in Section 3 has the form

$$
F(x, y, \lambda ; a)=\left(\begin{array}{c}
A(x, \lambda, z ; a) \\
y B(x, \lambda, z ; a)
\end{array}\right), \quad z=y^{2},
$$

where $a=(\alpha, \beta, \gamma) \in \mathbb{R}^{3}$ are unfolding parameters and $F(x, y, \lambda ; 0)=$ $G(x, y, \lambda)$. Here, $x$ and $y$ are bifurcation variables representing the amplitudes of the bifurcating modes and $\lambda$ is a distinguished bifurcation parameter. In the Brusselator, $x$ corresponds to an even and $y$ to an odd mode. The perturbed bifurcation diagrams associated with the unfolding $F$ are defined by the equation

$$
F(x, y, \lambda ; a)=0 .
$$

The solution set of (7.2) corresponding to $a=0$ is called the degenerate bifurcation diagram. In general, Eq. (7.2) possesses two types of solutions, namely, symmetric branches (under the reflection $y \rightarrow-y$ ) with $y=0$, determined by $A(x, \lambda, 0 ; a)=0$, and "asymmetric" branches determined by the simultaneous solutions of the equations $A=B=0$. We refer to the former as $S$-branches and to the latter as $H$-branches. Equations (7.1), (7.2) can be viewed as ordinary corank-1 bifurcations without symmetry in the Golubitsky-Schaeffer sense [5] coupled to $Z$ (2)-equivariant corank-1 bifurcations which are classified in [7] in the context of degenerate Hopf bifurcations. Each normal form has a specific interpretation in terms of these corank-1 bifurcations. Type (9) ${ }_{31}$ couples a pitchfork in the $S$-branch to a $H(2)$-bifurcation of [7], (8) ${ }_{221}$ a bifurcation point to a (2) $)_{\rho}$-bifurcation of $[1,4]$ which, in turn, organizes $H(2)$ and $H(3)$, whereas in the case of (6a) $)_{\rho, \kappa}$ a bifurcation point coalesces with a $H(7)$-point of [7].

The $S$-branches of types $(8)_{221}$ and (6a) $)_{p, \kappa}$ (see Sections 9 and 10) are essentially unfolded bifurcation points. [5]. There are alternative versions of these normal forms where the $S$-branch consists of an isola. For completeness we 
discuss in Section 11 the isola-version of $(8)_{221}$ and (6a) $)_{\rho, \kappa}$ although they do not appear in the Brusselator.

In Section 12 we illustrate how the generic type $(6)_{\rho}$ is organized by those codimension-3 bifurcations which occur in the Brusselator, including the further type $(7)_{2}$. This gives a more global picture of the bifurcating branches.

\subsection{Subordination}

The standard method $[1,5-7,11]$ for analyzing the perturbed bifurcation diagrams associated with the unfolding $F$ via (7.2) is to decompose the space $\mathbb{R}^{3}$ of unfolding parameters $(\alpha, \beta, \gamma)$ into connected open regions separated by varieties where the degeneracy conditions for codimension-1 bifurcations are satisfied.

We have to distinguish the following codimension-1 varieties,

$$
\begin{aligned}
& S H=\left\{a \mid y=0, A=A_{x}=A_{x x}=0\right\} \\
& S B=\left\{a \mid y=0, A=A_{x}=A_{\lambda}=0\right\} \\
& H B=\left\{a \mid A=B=0, A_{x} B_{\lambda}=A_{\lambda} B_{x}, A_{x} B_{z}=A_{z} B_{x}\right\} \\
& H(2)=\left\{a \mid y=0, A=B=0, A_{x} B_{\lambda}=A_{\lambda} B_{x}\right\} \\
& H(3)=\left\{a \mid y=0, A=B=0, A_{x} B_{z}=A_{z} B_{x}\right\} \\
& L H=\left\{a \mid y=0, A=B=A_{x}=0\right\} .
\end{aligned}
$$

The sets (7.3) have the following geometrical meaning. The first two, $S H$ and $S B$, correspond to a hysteresis and a bifurcation point in the $S$-branch, respectively [5]. The third, $H B$, yields a bifurcation point in the $H$-branch. The sets $H(2)$ and $H(3)$ are the $Z(2)$-symmetric codimension-1 bifurcations of Golubitsky and Langford [7]. On $H(2)$, the $H$ - and $S$-branches have a tangential contact and on $H(3)$ a limit point of the $H$-branch occurs for $y=0$. The last set, $L H$, corresponds to a corank-2 bifurcation, that is, a $H$-branch meets a limit point of an $S$-branch corresponding to the normal form $(1)_{21}$ of $[1,4]$. Normal forms for the degeneracies associated with $S H, S B, H B$ and $H(2), H(3)$ can be found in [5] and [7], respectively. In general, we have to consider one further set corresponding to a hysteresis in the $H$-branch which, however, does not appear in our normal forms.

Since we did not include non-degeneracy conditions in (7.3), the varieties $S H$, $S B$ etc. may contain subvarieties of codimension 1 (codimension 2 in $\mathbb{R}^{3}$ ) where a 
codimension-2 bifurcation takes place which are distinguished as follows,

$$
\begin{aligned}
& S P=\left\{a \mid y=0, A=A_{x}=A_{\lambda}=A_{x x}=0\right\} \\
& H(7)=\left\{a \mid y=0, A=B=0, A_{x} B_{\lambda}=A_{\lambda} B_{x}, A_{x} B_{z}=A_{z} B_{x}\right\} \\
& (1)_{31}=\left\{a \mid y=0, A=B=A_{x}=A_{x x}=0\right\} \\
& (1)_{22}=\left\{a \mid y=0, A=B=A_{x}=A_{z}=0\right\} \\
& (2)_{p}=\left\{a \mid y=0, A=B=A_{x}=B_{x}=0\right\} \\
& (6)_{p}=\left\{a \mid y=0, A=B=A_{x}=A_{\lambda}=0\right\} .
\end{aligned}
$$

Here, SP corresponds to the standard pitchfork (see [5]) and $H(7)$ to a degenerate $Z(2)$-symmetric corank-1 bifurcation defined in [7]. We may regard $H(7)$ as coalescing $H(2)$ and $H(3)$ bifurcations. The sets $(1)_{31}$ and (6) $)_{\rho}$ correspond to a $H$-branch meeting a hysteresis and a bifurcation point in the $S$-branch, respectively. On (1) $)_{22}$ and (2) $)_{\rho}$, respectively, a $H(3)$ and $H(2)$ bifurcation takes place at a limit point of the $S$-branch. For normal forms describing the latter corank-2 and codimension- 2 degeneracies see $[1,4]$.

Denoting the degenerate bifurcations corresponding to the sets in (7.3), (7.4) by the same symbol, the organisational power of the codimension-3 normal forms $(9)_{31},(8)_{221},(6 a)_{\rho, \kappa}$ are visualized in the subordination diagram of Fig. 1. In this diagram a degenerate bifurcation organizes a lower-codimension type if the latter can be reached from the former by following a sequence of arrows. We have included in Fig. 1 also the generic (codimension-0) bifurcations, namely, $S_{L}$ (limit point in the $S$-branch), $H_{L}$ (limit point in the $H$-branch) and $H(1)$ (generic $Z$ (2)-symmetric corank-1 bifurcation, see [7]).

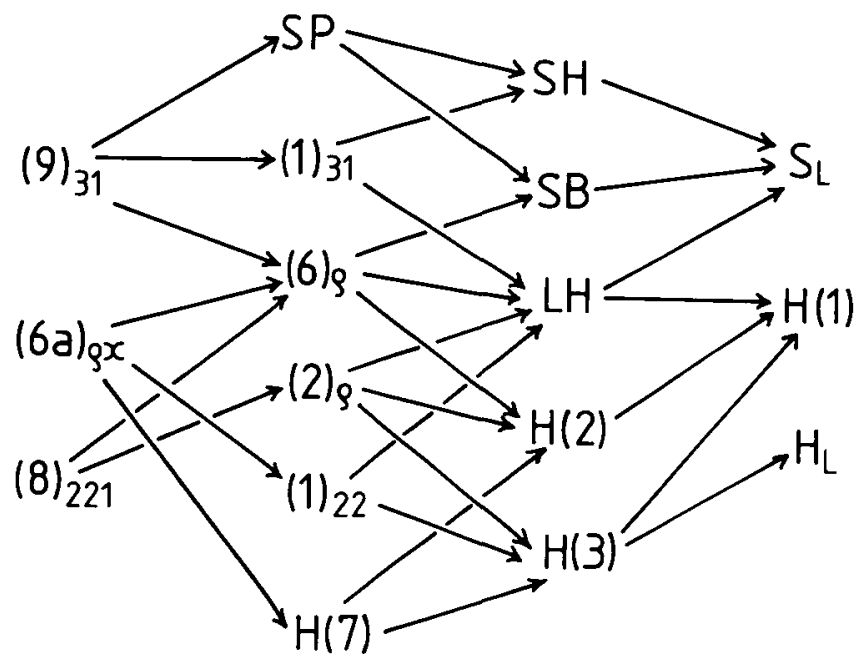

Figure 1. Subordination diagram 


\subsection{Stability of the bifurcating branches}

To any non-degenerate solution of (7.2) we associate two stability symbols $( \pm, \pm)$ corresponding to the signs of the real parts of the eigenvalues of the Jacobean of $F$, evaluated at the solution. If (7.2) is regarded as an equation for the steady states of a dynamical system

$$
\frac{d}{d t}\left(\begin{array}{l}
x \\
y
\end{array}\right)=\left(\begin{array}{c}
A\left(x, \lambda, y^{2}, a\right) \\
y B\left(x, \lambda, y^{2}, a\right)
\end{array}\right)
$$

a solution of (7.2) corresponds to a stable steady state only if the stability symbol is $(-,-)$. By varying $\lambda$, one sign in the stability symbol is reversed when crossing a limit point or a $H(1)$-point (secondary bifurcation). The contact equivalence of Section 3 has been chosen to preserve these stability exchanges. In [11] it has been shown that the stability exchanges are also preserved if the solutions of (7.2) correspond to the steady state branches of the Brusselator near a double eigenvalue, where $\left.F\right|_{a=0}$ is contact equivalent to the bifurcation problem obtained from the reaction diffusion equation of the Brusselator via Liapunov-Schmidt reduction.

In the diagrams of Sections 8-11 we use the letters $s, u, n$ to denote the stability symbols $(-,-),(+,+),(+,-)$, respectively.

\section{Perturbed bifurcation diagrams for type $(9)_{31}$}

Choosing signs $\varepsilon_{1}=\varepsilon_{2}=-1, \varepsilon^{\prime}=1$, the universal unfolding of the normal form (9) ${ }_{31}$ is given by [4]

$$
F=\left(\begin{array}{c}
-\left(x^{3}-x \lambda+\varepsilon z+\alpha+\beta x^{2}\right) \\
-y(x-\lambda+\gamma)
\end{array}\right), \quad \varepsilon= \pm 1
$$

In the Brusselator only the case $\epsilon=1$ occurs but it is very easy to discuss both signs $\varepsilon= \pm 1$ simultaneously.

From (7.3) and Fig. 1 the codimension-1 sets of $F$ are obtained as follows.

$L H$ is given parametrically in the form

$$
\gamma=-x+2 \beta x+3 x^{2}, \quad \alpha=2 x^{3}+\beta x^{2} .
$$


Solving the first equation of (8.2) for $x$ and inserting into the second yields $\alpha$ as a smooth function of $(\beta, \gamma)$,

$$
L H: \alpha=\alpha(\beta, \gamma)=\beta \gamma^{2}-2 \gamma^{3}+\gamma^{2} O(2),
$$

where the symbol $O(j)$ denotes terms of at least $j$-th order in the unfolding parameters.

A parametric representation for $H(2)$ is

$$
\gamma=2 x-2 \beta x-3 x^{2}, \quad \alpha=-x^{2}(1-2 x-\beta) .
$$

Proceeding as with $L H$ we obtain an explicit representation $\alpha$ as smooth function of $(\beta, \gamma)$,

$$
H(2): \alpha=\alpha(\beta, \gamma)=-\frac{1}{4} \gamma^{2}(1+O(1))
$$

The sets $S H$ and $S B$ have the same form as the standard pitchfork of [5],

$$
S H: \alpha=\beta^{3} / 27, \quad S B: \alpha=0 .
$$

The codimension- 2 sets are given by

$$
\begin{array}{ll}
S P: & \alpha=\beta=0 \\
(1)_{31}: & \alpha=\beta^{3} / 27, \quad \gamma=\frac{1}{3}\left(\beta-\beta^{2}\right) \\
(6)_{\rho}: & \alpha=\gamma=0 .
\end{array}
$$

It may now be verified that the decomposition of the unfolding space $\mathbb{R}^{3}$ via $L H \cup H(2) \cup S H \cup S B$ is as shown in Fig. 2 for sections $\beta=$ constant. Projections of the perturbed bifurcation diagrams onto the $(x, \lambda)$-plane are shown in Fig. 3 for the regions marked in Fig. 2. Since the $H$-branches do not possess limit points with $y \neq 0$, the $(x, \lambda)$-projections contain complete information about the

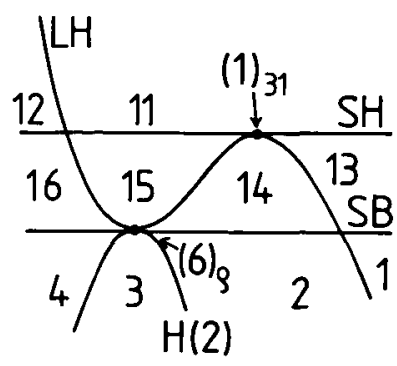

$\beta>0$

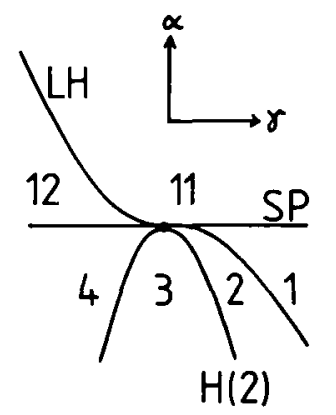

$\beta=0$

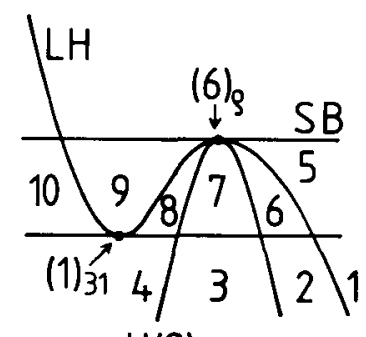

$H(2)$

$\beta<0$

Figure 2. Decomposition of unfolding space for type $(9)_{31}$ 
qualitative behaviour of all branches. To illustrate this we have sketched in Fig. 4 a three-dimensional diagram corresponding to region 4 in Fig. 2.

Observe the stability exchanges $(+,+) \rightarrow(-,-)$ in some of the diagrams of Fig. 3 corresponding to a Hopf bifurcation. Although these exchanges are not preserved by contact equivalence, there is topological evidence where at least one, respectively an odd number of Hopf bifurcations must be present.
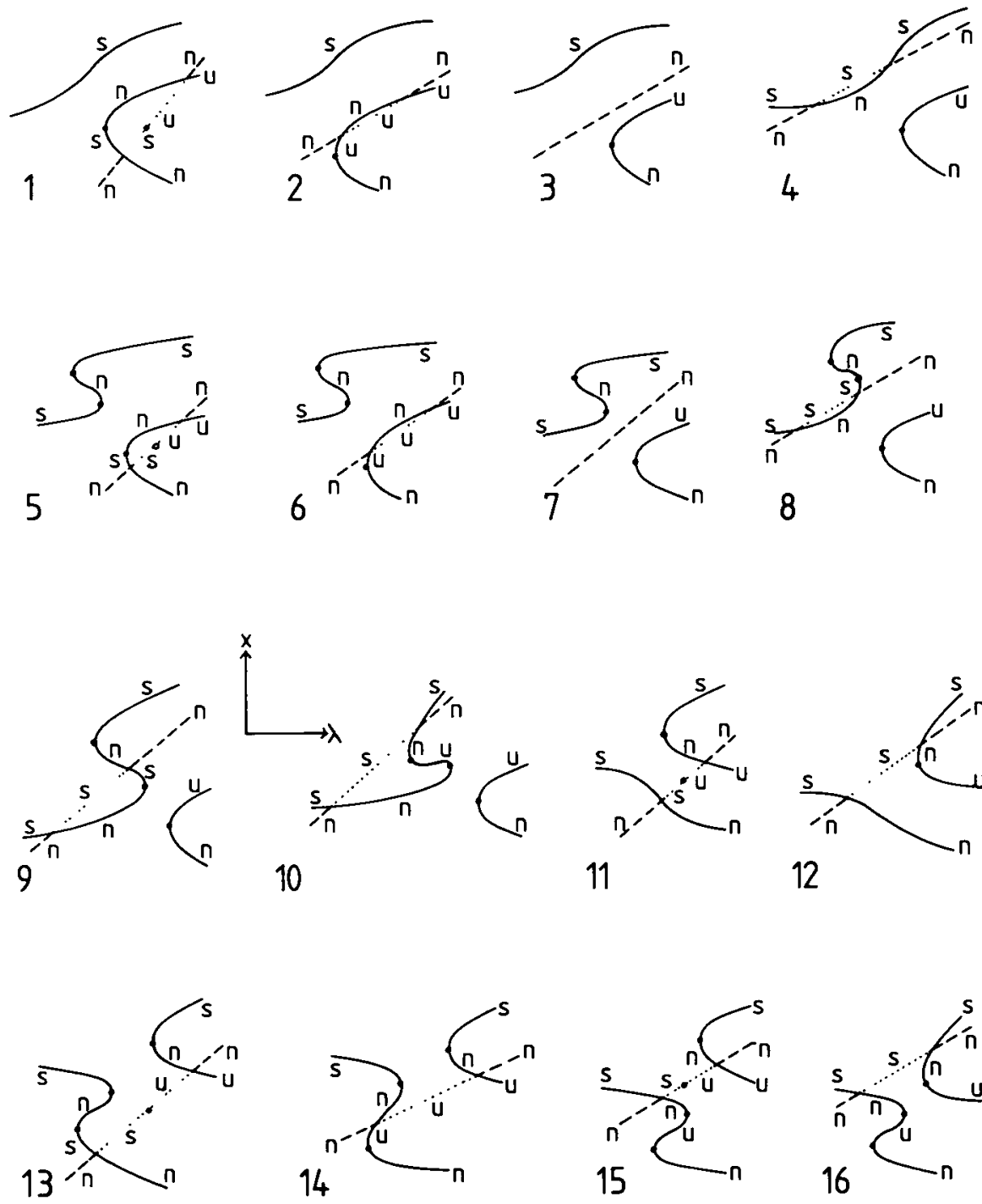

10
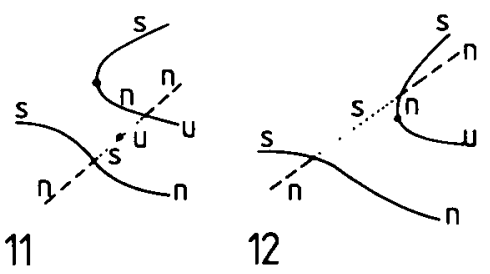

Figure 3. Projections of bifurcation diagrams onto $(x, \lambda)$-plane for parameters in the regions marked in Figure 2. Solid lines are $S$-branches, broken (dotted) lines are $H$-branches for $\varepsilon=1$ $(\varepsilon=-1)$. 


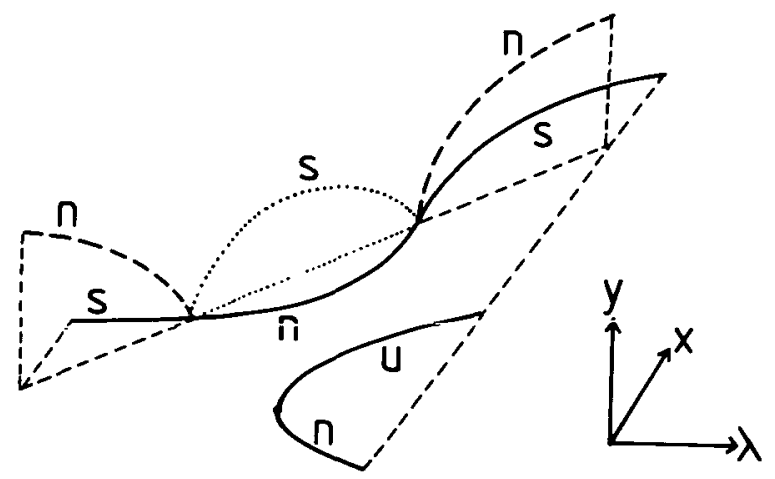

Figure 4. Three-dimensional version $(y \geqslant 0)$ of diagram 4 in Fig. 3.

\section{Perturbed bifurcation diagrams for type $(8)_{221}$}

Here,

$$
F=\left(\begin{array}{c}
-\left(x^{2}-\lambda^{2}+\varepsilon z+\alpha\right) \\
-y\left(x^{2}-\lambda+\beta+\gamma x\right)
\end{array}\right), \quad \varepsilon= \pm 1,
$$

where the sign $\varepsilon=-1$ occurs in the Brusselator. The relevant codimension- 1 sets are (cf. Fig. 1 and (7.3)),

$$
\begin{array}{ll}
S B: & \alpha=0 \\
L H: & \alpha=\beta^{2} \\
H(3): & \alpha=\beta^{2}-\frac{1}{4} \gamma^{2}\left(1+2 \beta-\gamma^{2} / 4\right),
\end{array}
$$
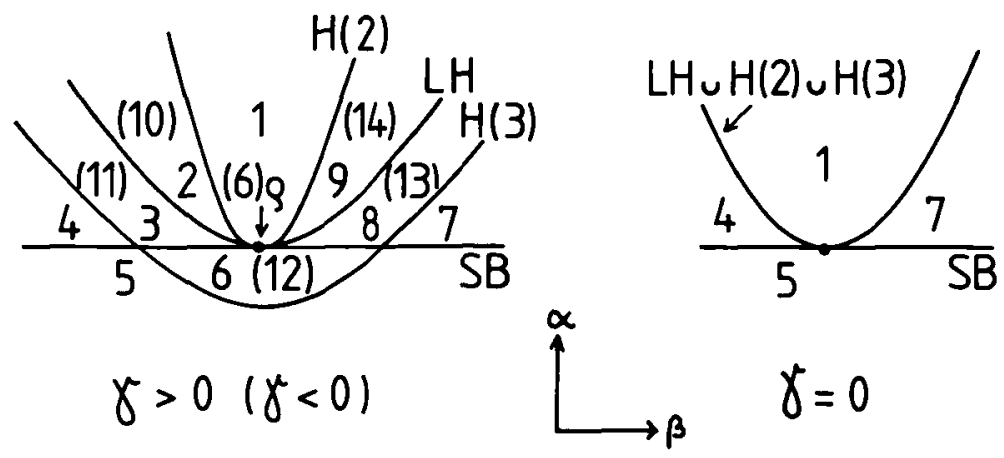

Figure 5. Decomposition of unfolding space for type (8) ${ }_{221}$. The numbers in brackets refer to $\gamma<0$. 

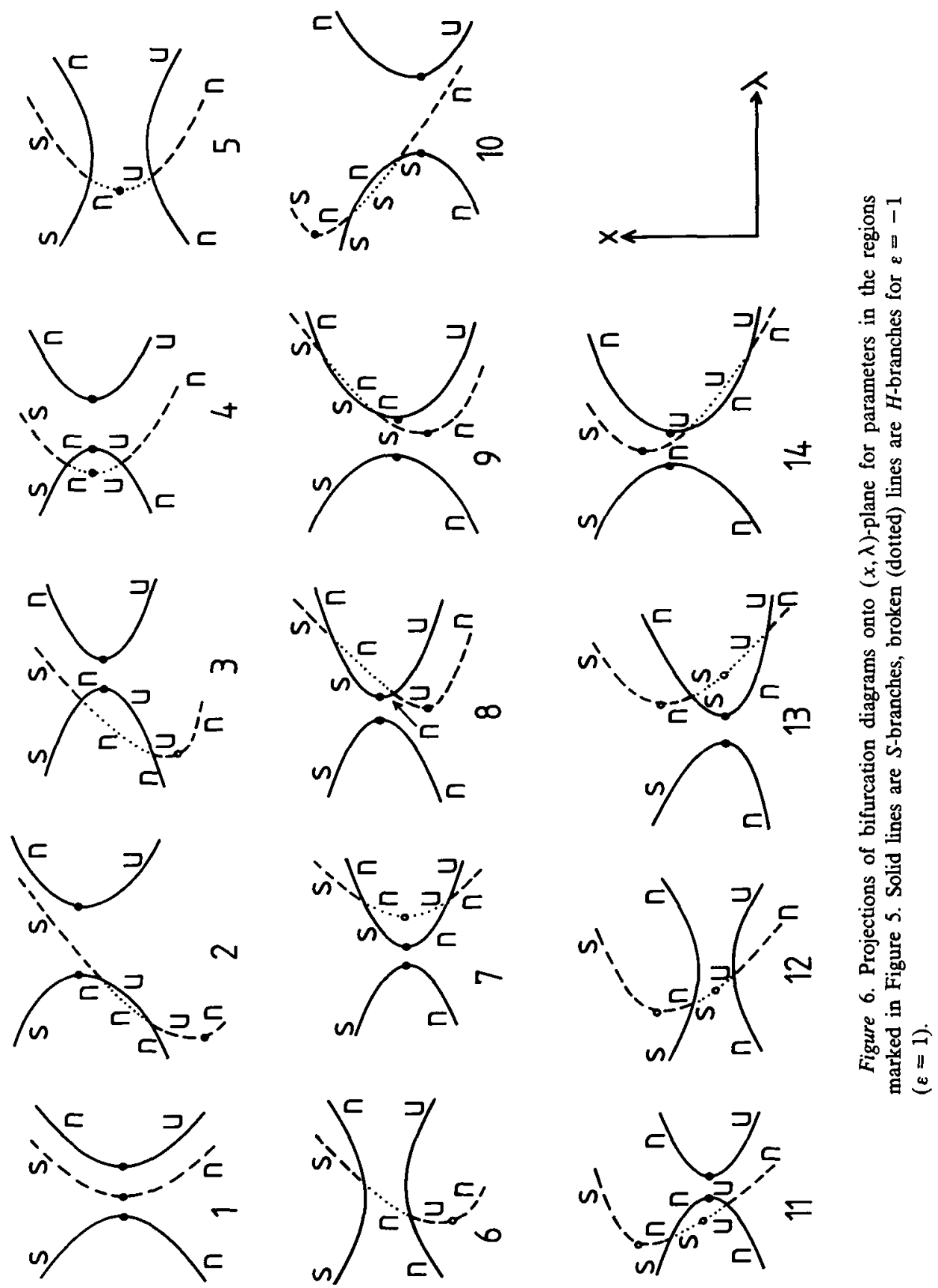


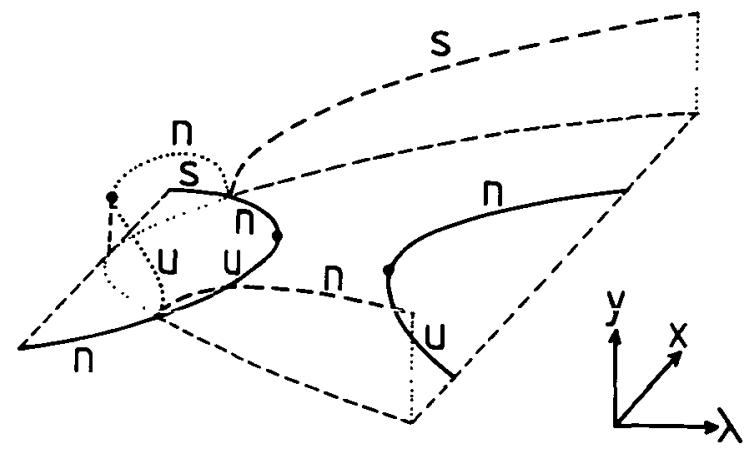

Figure 7. Three-dimensional version $(y \geqslant 0)$ of diagram 4 in Figure 6.

and $H(2)$ is implicitly determined by

$$
\begin{aligned}
& \lambda(2 x+\gamma)-x=0 \\
& \beta+\gamma x+x^{2}-\lambda=0 \\
& \alpha=\lambda^{2}-x^{2} .
\end{aligned}
$$

Solving (9.5a, b) for $x$ and $\lambda$ in terms of $(\beta, \gamma)$ and substituting the result into (9.5c) yields $\alpha$ as smooth function of $(\beta, \gamma)$

$$
H(2): \alpha=\alpha(\beta, \gamma)=\beta^{2}+\beta^{2} \gamma^{2}(1+O(1)) .
$$

The codimension- 2 sets are

$$
\begin{aligned}
& (6)_{\rho}: \alpha=\beta=0 \\
& (2)_{\rho}: \alpha=\beta^{2} .
\end{aligned}
$$

The decomposition of unfolding space by $L H \cup S B \cup H(2) \cup H(3)$ is shown in Fig. 5 for sections $\gamma=$ const. Projections of the perturbed bifurcation diagrams onto the $(x, \lambda)$-plane are collected in Fig. 6 Since the projection of the $H$-branches with $y \geqslant 0$ onto $(x, \lambda)$-plane is one-to-one and preserves limit points, the $(x, \lambda)$-diagrams of Fig. 6 contain complete information about the qualitative behaviour of all branches. In Fig. 7 a three-dimensional diagram corresponding to region 4 in Fig. 5 is sketched.

\section{Perturbed bifurcation diagrams for type $(6 a)_{\rho, k}$}

The universal unfolding of the normal form $(6 a)_{\rho, \kappa}$ is given by

$$
F=\left(\begin{array}{c}
\varepsilon_{1}\left(x^{2}-\lambda^{2}+\varepsilon z^{2}+2 \kappa z \lambda+\alpha+\beta z\right) \\
\varepsilon_{2} y(x-\rho \lambda-\gamma)
\end{array}\right),
$$

where

$$
\varepsilon_{1}, \varepsilon_{2}, \varepsilon= \pm 1, \rho \geqslant 0
$$



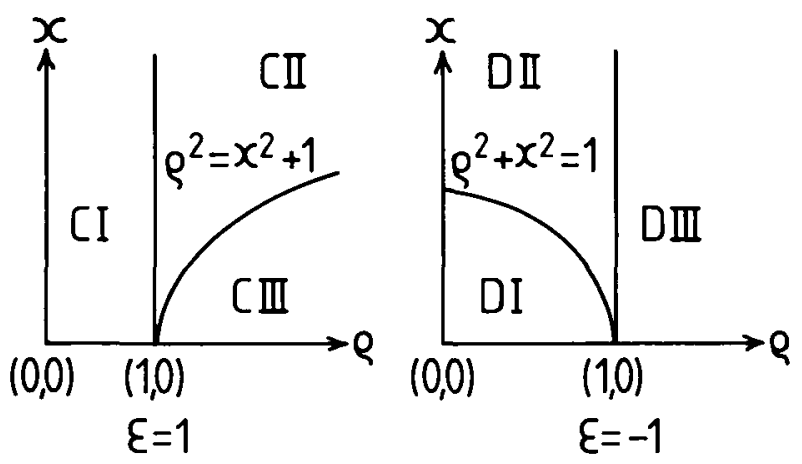

Figure 8 . The six modal cases of $(6 a)_{p, \kappa}$.

and

$$
\rho \neq 1, \varepsilon\left(\rho^{2}-1\right) \neq \kappa^{2} .
$$

We may confine to $\kappa \geqslant 0$ because the reversal $\lambda \rightarrow-\lambda$ and an appropriate contact transformation brings (10.1) to a form where $\kappa$ is replaced by $-\kappa$. The presence of the modal parameters $\rho, \kappa$ implies that $(6 a)_{\rho, \kappa}$ has codimension $5^{* *}$, that is, topological codimension 3 . In virtue of the inequalities $(10.2 b)$ we have to distinguish six different modal cases for $(6 \mathrm{a})_{\rho, \kappa}$ which are denoted by $C I, I I, I I I$, $D I, I I, I I I$. The regions in the $(\rho, \kappa)$-plane to which these cases correspond are shown in Fig. 8. We discuss perturbed bifurcation diagrams associated to (10.1) with the signs $\varepsilon_{1}=\varepsilon_{2}=1$ for the cases $C I, D I, D I I$ and with $\varepsilon_{1}=\varepsilon_{2}=-1$ for $C I I, C I I I, D I I I$. Recall from Section 3 that the cases $C I, D I, D I I$ and $D I I I$ occur in the Brusselator with signs $\varepsilon_{1}=\varepsilon_{2}=1$ and $\varepsilon_{1}=\varepsilon_{2}=-1$, respectively.

For fixed $\gamma$ we define the straight line $L$ in the $(x, \lambda)$-plane by $B=0$, that is,

$$
L: x=\rho \lambda+\gamma \text {. }
$$

Since the $H$-branches are located in the plane $E$ spanned by $L$ and the $y$-axis, any perturbed bifurcation diagram can be decomposed into the $H$-branch lying in $E$ and the $S$-branch and $L$ lying in the $(x, \lambda)$-plane. In Fig. 9 this is shown for the unperturbed diagrams (parameters equal to zero). From Fig. 9 we infer the geometrical meaning of the six sub-types $C I, C I I$ etc. The cases $C I, D I, D I I$ are distinguished from $C I I, C I I I, D I I I$ by the position of $L$ relative to the unperturbed $S$-branch (Fig. 9(a)). Modulo orientations, the unperturbed $H$-branches can be grouped into three different topological types, namely, $(C I, D I I I),(C I I$, $D I I)$ and $(C I I I, D I)$ (Fig. 9(b)) corresponding to the three different modal cases of the corank-1 $Z(2)$-bifurcation $H(7)$ [7] (apart from stabilities). The distinction of the six sub-types of $(6 a)_{\rho, \kappa}$ is obtained by combining the three cases of Fig. 9(b) with Fig. 9(a). We note that Fig. 9(a) corresponds-modulo stabilities and orientations - to the two different modal cases of type $(6)_{\rho}$ (see $\left.[1,11]\right)$. 


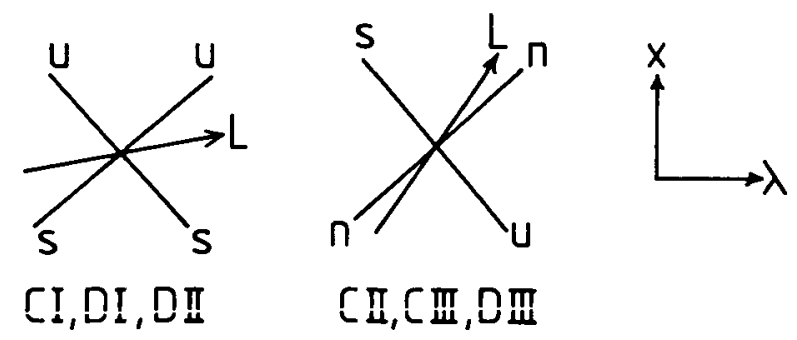

(a)

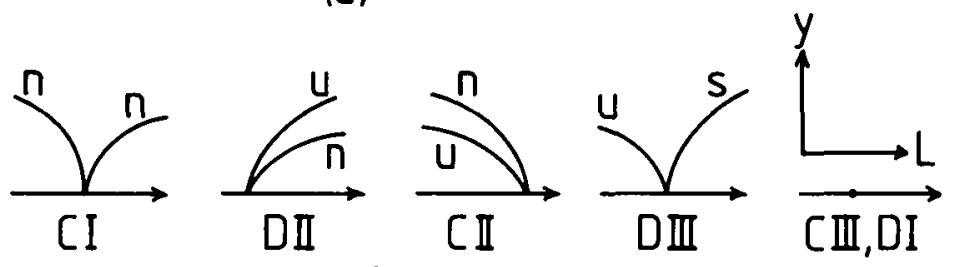

(b)

Figure 9. Unperturbed bifurcation diagrams associated with type (6a) ${ }_{p, \kappa}$ (a) $S$-branches. (b) $H$-branches.

For analysing the varieties in the unfolding space it is convenient to introduce transformed unfolding parameters $(\bar{\alpha}, \bar{\beta})$ as follows,

$$
\begin{aligned}
& \bar{\alpha}=\left(\rho^{2}-1\right) \alpha-\gamma^{2} \\
& \bar{\beta}=\left\{\kappa \rho \gamma+\frac{1}{2}\left(1-\rho^{2}\right) \beta\right\} \operatorname{sgn} \Delta,
\end{aligned}
$$

where

$$
\Delta=\varepsilon\left(\rho^{2}-1\right)+\kappa^{2} .
$$

Assuming $\kappa \rho \neq 0$ it is straightforward to derive analytic expressions for the codimension-1 and -2 sets of Section 7 . In terms of the parameters $(\bar{\alpha}, \bar{\beta}, \gamma)$ they take the simple forms,

$$
\begin{array}{ll}
S B: & \bar{\alpha}=-\gamma^{2} \\
H(2): & \bar{\alpha}=0 \\
H(3): & \bar{\alpha}=-\bar{\beta}^{2} / \kappa^{2} \\
L H: & \bar{\alpha}=-\gamma^{2} / \rho^{2} \\
H B: & \bar{\alpha}=\bar{\beta}^{2} / \Delta, \bar{\beta}>0,
\end{array}
$$

and

$$
\begin{array}{ll}
H(7): & \bar{\alpha}=\bar{\beta}=0 \\
(1)_{22}: & \bar{\alpha}=-\gamma^{2} / \rho^{2}, \bar{\beta}=(\kappa \gamma / \rho) \operatorname{sgn} \Delta \\
(6)_{\rho}: & \bar{\alpha}=\gamma=0 .
\end{array}
$$


The varieties (10.6) are shown in Fig. 10 for sections $\bar{\beta} \neq 0$. In Fig. 10(a) the sets $H(2), S B$ and $L H$ are sketched. The sets $H(2), H B$ and $H(3)$ are presented in Fig. 10(b) for $\bar{\beta}>0$ and in Fig. 10(c) for $\bar{\beta}<0$. The open regions in unfolding space separated by the sets $(10.6)$ are numbered by triples $(i, j)(1 \leqslant i \leqslant 6$, $1 \leqslant j \leqslant 5$ ) corresponding to the intersection of region $i$ in Fig. 10(a) and region $j$ in Figs. 10(b), (c).

The perturbed bifurcation diagrams corresponding to the regions $(i, j)$ are summarized in Fig. 11. We have used the same representation as in Fig. 9, that is, the diagrams are decomposed into $S$-branches and the line $L$ in the $(x, \lambda)$-plane (Fig. 11(a)) and $H$-branches lying in the plane $E$ spanned by $L$ and the $y$-axis (Fig. 11(b)) corresponding to the regions $1-6$ in Fig. 10(a) and regions $1-5$ in
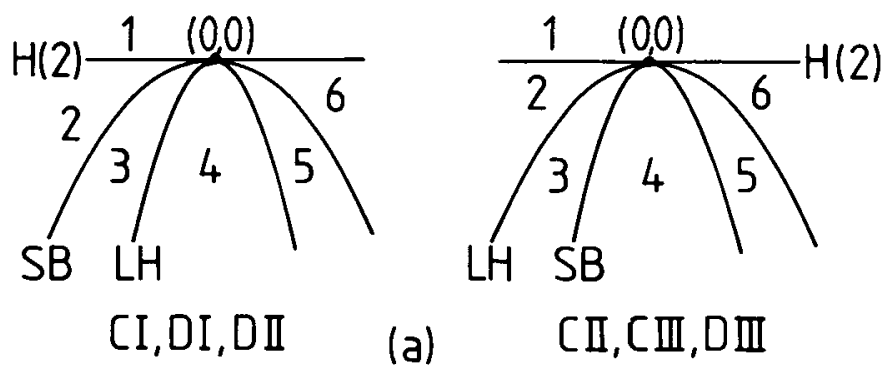

(a)

CII,CII,DII
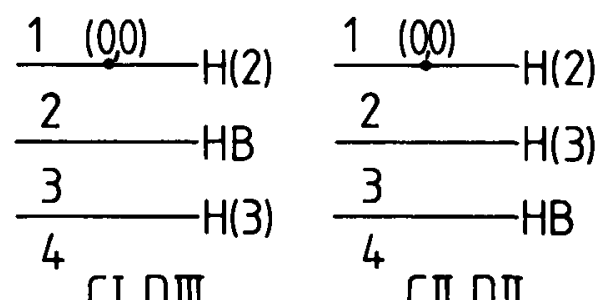

(b) $\bar{\beta}>0$
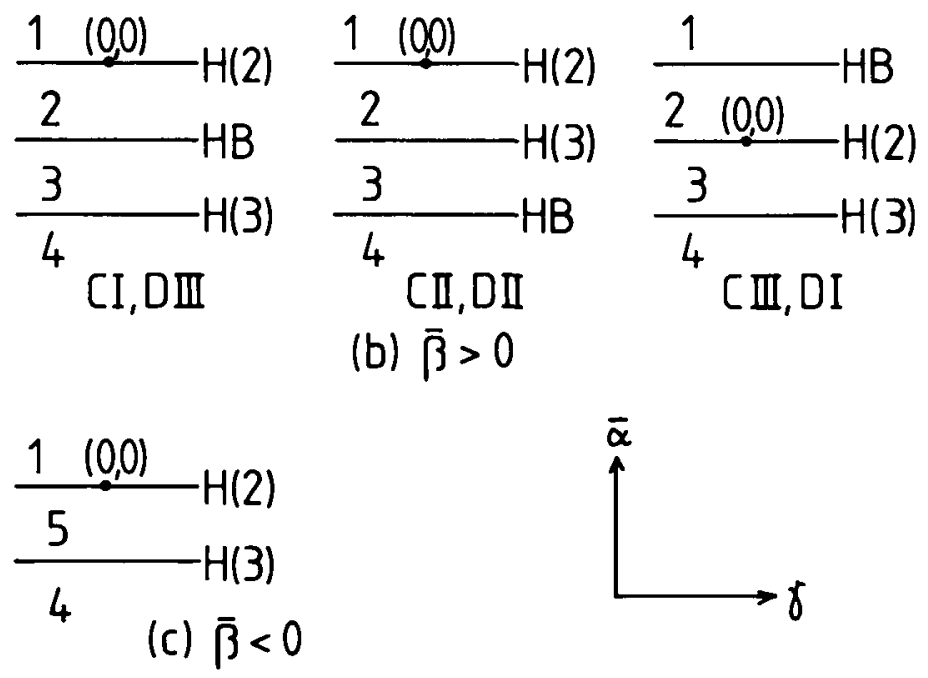

Figure 10. Sections $\bar{\beta} \neq 0$ of the varieties (4.6) for type (6a) $)_{\rho, \kappa^{-}}$(a) The sets $H(2), S B, L H$. The sets $H(2), H B, H(3)$ are shown in Figs. 10(b) and (c) for $\bar{\beta}>0$ and $\bar{\beta}<0$, respectively. The unfolding space is decomposed into open regions $(i, j)$ obtained by intersection of region $i$ in (a) and region $j$ in (b) or (c). 
524

Gerhard Dangelmayr

[39]
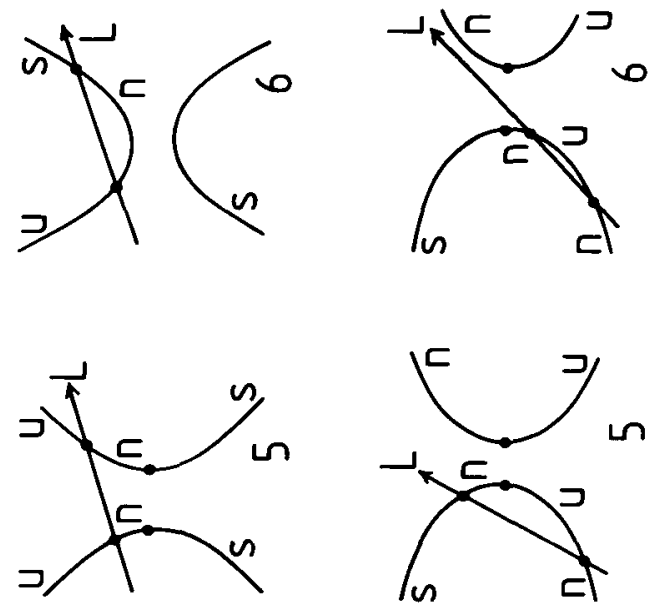

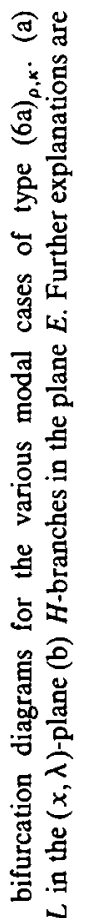
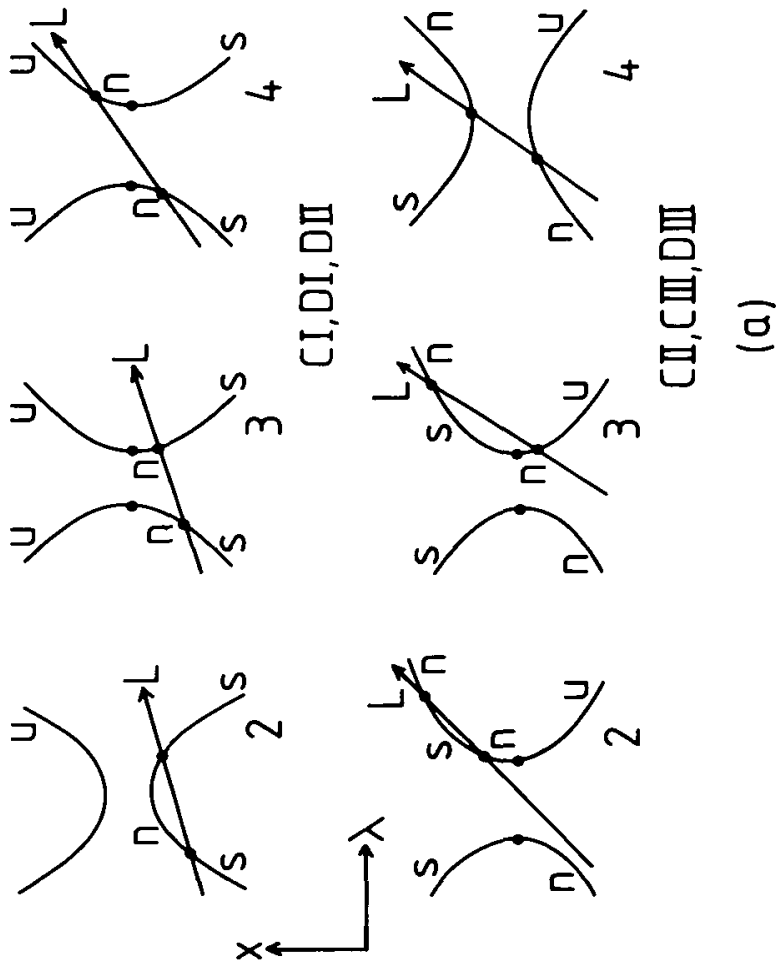

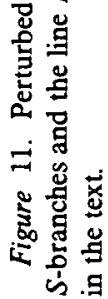

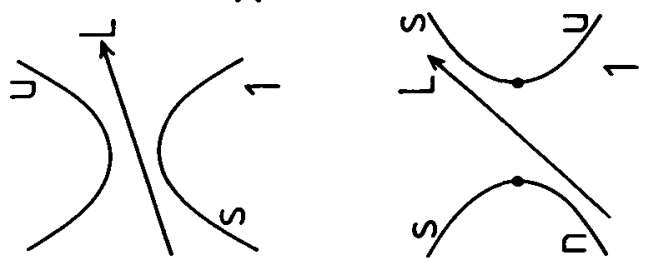

https://doi.org/10.1017/S0334270000005555 Published online by Cambridge University Press 


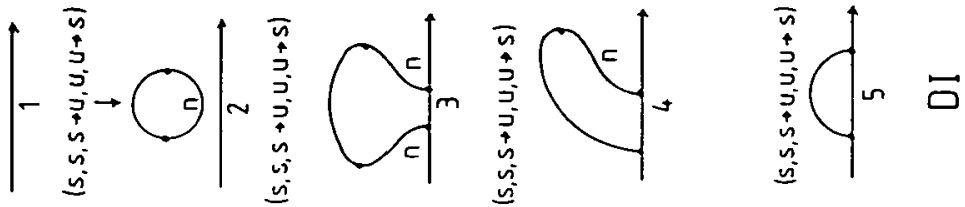

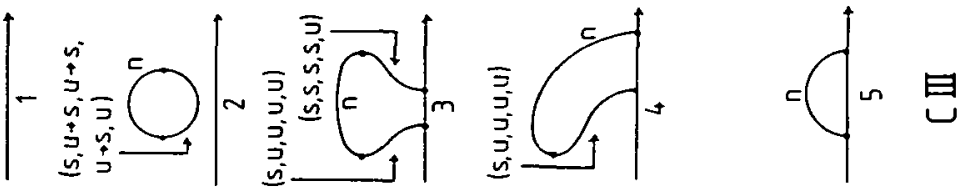

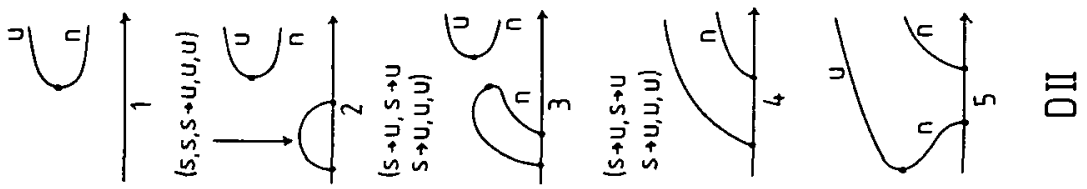

(c)

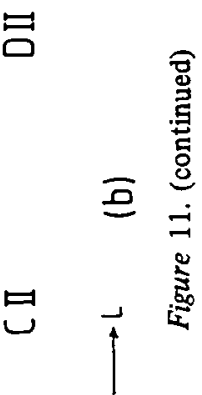

$3 \mid-5=$
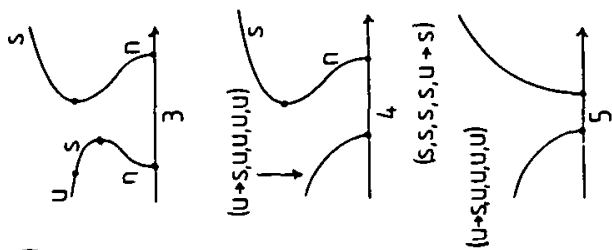

붐

$i^{1}$
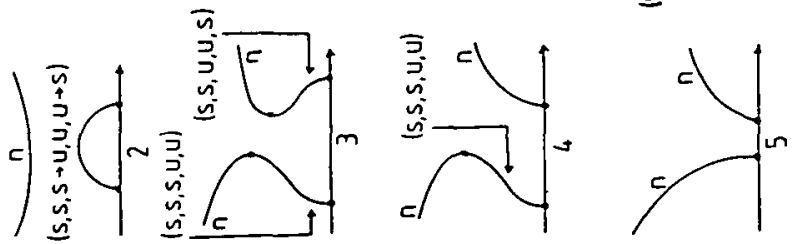

$\varpi$ 


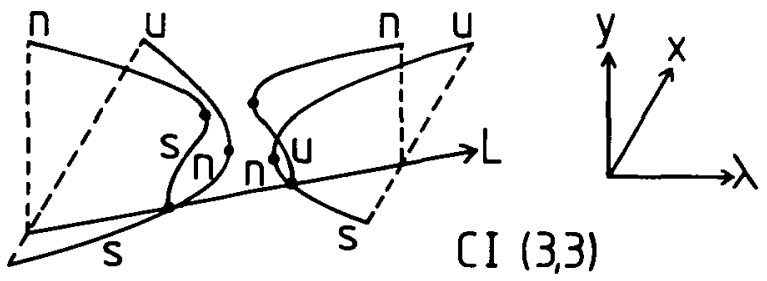

Figure 12 . Three-dimensional perturbed bicfurcation diagram corresponding to region $(3,3)$ for the modal case $C I$ of type $(6 a)_{p, \kappa}$.

Figs. 10(b), (c), respectively. The two points where the line $L$ intersects the $S$-branch are secondary bifurcation points. At these points an $H$-branch emerges from the $S$-branch as shown in Fig. 11(b). The perturbed bifurcation diagram corresponding to a non-empty region $(i, j)$ is obtained by putting diagram $j$ in Fig. 11(b) vertically onto diagram $i$ in Fig. 11(a). The stability symbols in the brackets associated with a sub-branch of an $H$-branch in Fig. 11(b) correspond, respectively, to the regions $(2,3,4,5,6)$ of Fig. 10(a). The symbols $s \rightarrow u$ and $u \rightarrow s$ indicate a stability exchange $s$ to $u$ and $u$ to $s$ via a tertiary bifurcation, respectively, when moving along $L$ from left to right. In order to illustrate how the diagrams of Figs. 11(a) and (b) are combined we have sketched the three-dimensional perturbed bifurcation diagram for case $C I$, region $(3,3)$, in Fig. 12 .

\section{Isola-versions of types $(8)_{221}$ and $(6 a)_{p, \kappa}$}

In this Section we analyze the perturbed bifurcation diagrams for the isola-versions of the types (8) 221 and (6a) $)_{\rho, \kappa}$ which are obtained by replacing $x^{2}-\lambda^{2}$ in (3.1) and (4.1) by $x^{2}+\lambda^{2}$.

\subsection{Type (8) ${ }_{221}$-isola}

Proceeding as in Section 9 we find the following expressions for the codimension- 1 and -2 sets,

$$
\begin{array}{ll}
S B: & \alpha=0 \\
L H: & \alpha=-\beta^{2} \\
H(3): & \alpha=-\beta^{2}-\frac{1}{4} \gamma^{2}\left(1-2 \beta+\frac{1}{4} \gamma^{2}\right), \\
H(2): & \alpha=-\beta^{2}+\beta^{2} \gamma^{2}(1+O(1))
\end{array}
$$


and

$$
\begin{array}{ll}
(6)_{\rho}: & \alpha=\beta=0 \\
(2)_{\rho}: & \alpha=-\beta^{2} .
\end{array}
$$

The decomposition of unfolding space is shown in Fig. 13 for sections $\gamma \neq 0$. Perturbed bifurcation diagrams corresponding to the regions of Fig. 13 are summarized in Fig. 14.

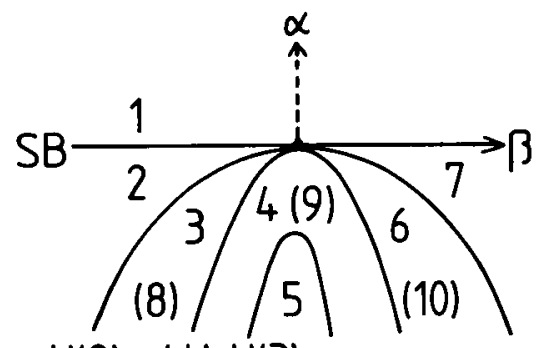

$H(2) \quad L H H(3)$

$$
\gamma>0 \quad(\gamma<0)
$$

Figure 13. Decomposition of unfolding space for type (8) ${ }_{221}$-isola. The numbers in brackets refer to $\gamma<0$.
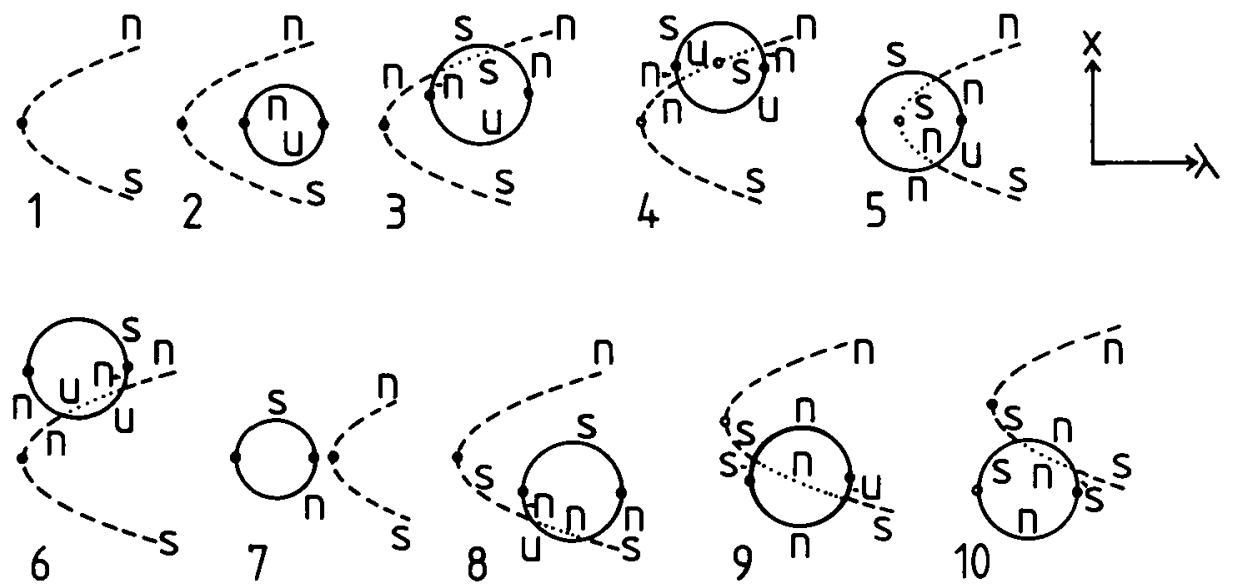

Figure 14. Projections of bifurcation diagrams onto $(x, \lambda)$-plane for parameters in the regions marked in Figure 13. Meaning of solid, broken and dotted lines as in Figure 6. 


\subsection{Type $(6 a)_{p, k}$-isola}

Defining $\Delta$ by

$$
\Delta=\varepsilon\left(1+\rho^{2}\right)-\kappa^{2},
$$

we have to distinguish thine modal cases as follows,

$$
\begin{array}{ll}
A: & \varepsilon=-1 \\
B I: & \varepsilon=1, \Delta>0 \\
B I I: & \varepsilon=1, \Delta<0 .
\end{array}
$$

For all three cases of (11.4) we choose the signs $\varepsilon_{1}=\varepsilon_{2}=-1$. The unperturbed $S$-branch consists of the origin which blows up to a circle by unfolding. The unperturbed $H$-branches for the cases $A$ and $B I I$ have the form of Fig. 9(b), case $D I I I$ (with $u$ and $s$ interchanged) and case $C I I$ (with $u$ replaced by $s$ ), respectively, where the straight line $L$ is defined by (10.3) as in Section 10 . The unperturbed $H$-branch for the case $B I$ consists of the origin.

Defining

$$
\begin{aligned}
& \bar{\alpha}=\left(1+\rho^{2}\right) \alpha+\gamma^{2} \\
& \bar{\beta}=\left\{\kappa \rho \gamma-\frac{1}{2}\left(1+\rho^{2}\right) \beta\right\} \operatorname{sgn} \Delta,
\end{aligned}
$$

the sets $S B$ and (1) 22 are given by

$$
\begin{array}{ll}
S B: & \bar{\alpha}=\gamma^{2} \\
(1)_{22}: & \bar{\alpha}=-\gamma^{2} / \rho^{2}, \bar{\beta}=-(\kappa \gamma / \rho) \operatorname{sgn} \Delta .
\end{array}
$$

The analytic expressions for the remaining codimension- 1 and -2 sets are the same as in Section 10 (Eqs. (10.6), (10.7)). In Fig. 15 the sets $H(2), S B$ and $L H$ are shown in the $(\bar{\alpha}, \gamma)$-plane. The sets $H(2), H B$ and $H(3)$ have the same form as in

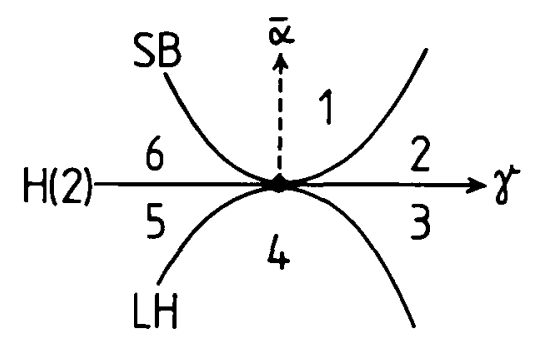

Figure 15. The sets $H(2), S B$ and $L H$ for type $(6 a)_{\rho, k}$-isola. 

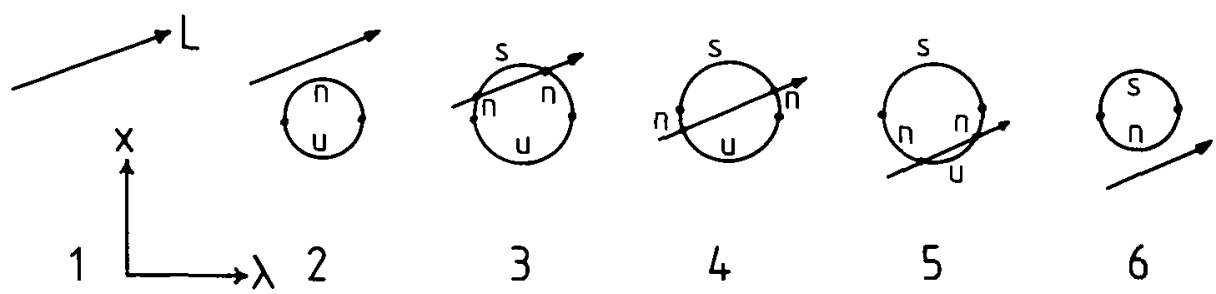

4

5

6

(a)
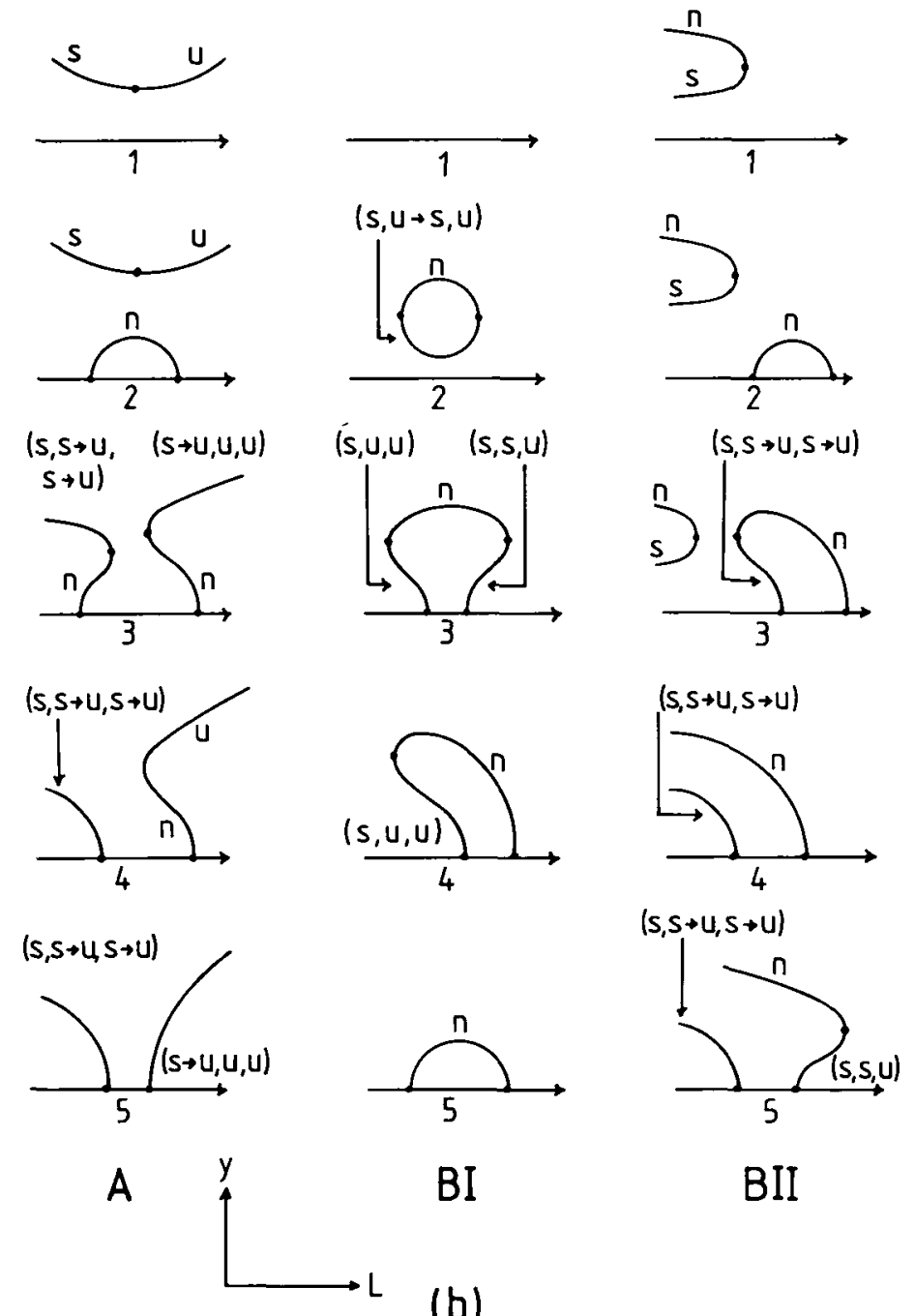

BII

Figure 16. Perturbed bifurcation diagrams for type $(6 a)_{\rho, \kappa}$-isola. 
Figs. 10(b), (c), for sections $\bar{\beta} \neq 0$. The unfolding space is decomposed into regions $(i, j)$ obtained by intersection of region $i$ in Fig. 13 and region $j$ in Figs. 10(b), (c). The perturbed bifurcation diagrams are summarized in Fig. 16 which must be interpreted similarly as Fig. 11 . The stability symbols in the brackets in Fig. 16(b) refer, respectively, to the regions $(3,4,5)$ of Fig. 15.

\section{Subordination of type (6),}

The objective of this Section is to demonstrate how the "generic" bifurcation of type (6) $)_{\rho}$ is organized by those codimension-3 bifurcations which occur in the Brusselator. The results are summarized in Fig. 17 for the types $(9)_{31},(8)_{221}$ and the further type (7) ${ }_{2}$ (see [1, 4], in [1] the subscript 2 is suppressed), and in Fig. 18 for the relevant modal cases of type $(6 a)_{p, \kappa}$. We have not analysed type $(7)_{2}$ in this paper because it is discussed in detail in [1]. From the results of [11] it follows that the degeneracy conditions for type $(7)_{2}$ are satisfied for the Brusselator on certain lines in parameter space (the non-degeneracy conditions still need to be proved). The essential feature of the degenerate bifurcation diagram of $(7)_{2}$ is that the $H$-branch terminates tangentially in the $S$-branch where the $(y, \lambda)$-projection encounters a cusp. The cases $k>0$ and $k<0$ give rise to different signs in the normal form $(7)_{2}$. Recall from Section 3 that the modal cases $D I I I ; D I, I I$ and $D I, D I I, C I$ of type (6a) $)_{\rho, \kappa}$ occur, respectively, if $k>0, k \leqslant-4$ and $k=-2$.

The letter $T$ in Figs. 17, 18 refers to the solution branch corresponding to the trivial (spatially and temporally homogeneous) solution of the reaction diffusion equations associated with the Brusselator model. For each type and modal case Figs. 17 and 18 show the degenerate diagram in the middle and the two different subordinate modal cases of (6) $)_{\rho}$ to the left and right of the degenerate diagram. We have used the notation of [11] to denote the various $(6)_{p}$-cases. The Roman numeral indicates the number of solutions emanating from the trivial solution at the bifurcation point and the subscript denotes the number of them which are stable. Note that each $H$-branch in Figs. 17, 18 corresponds actually to two branches via reflection $y \rightarrow-y$. 


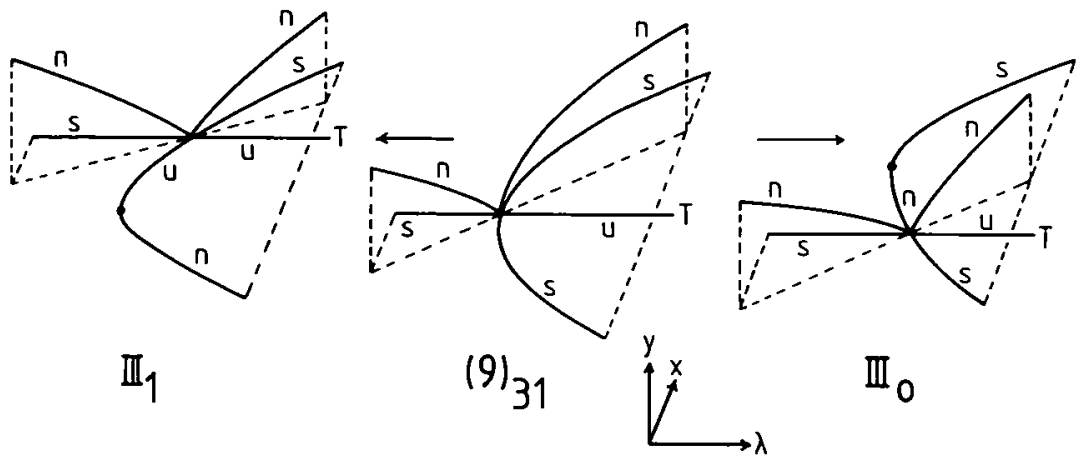

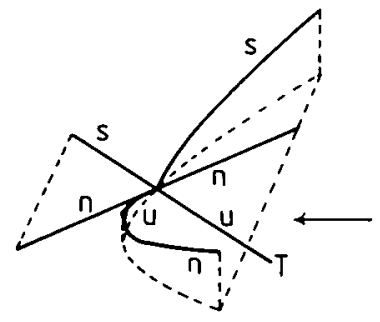

$\mathbb{I}_{2}$

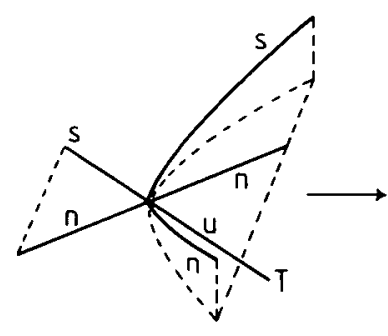

(8) 221

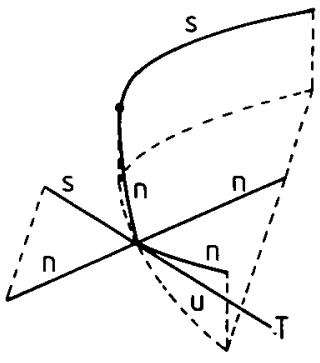

III
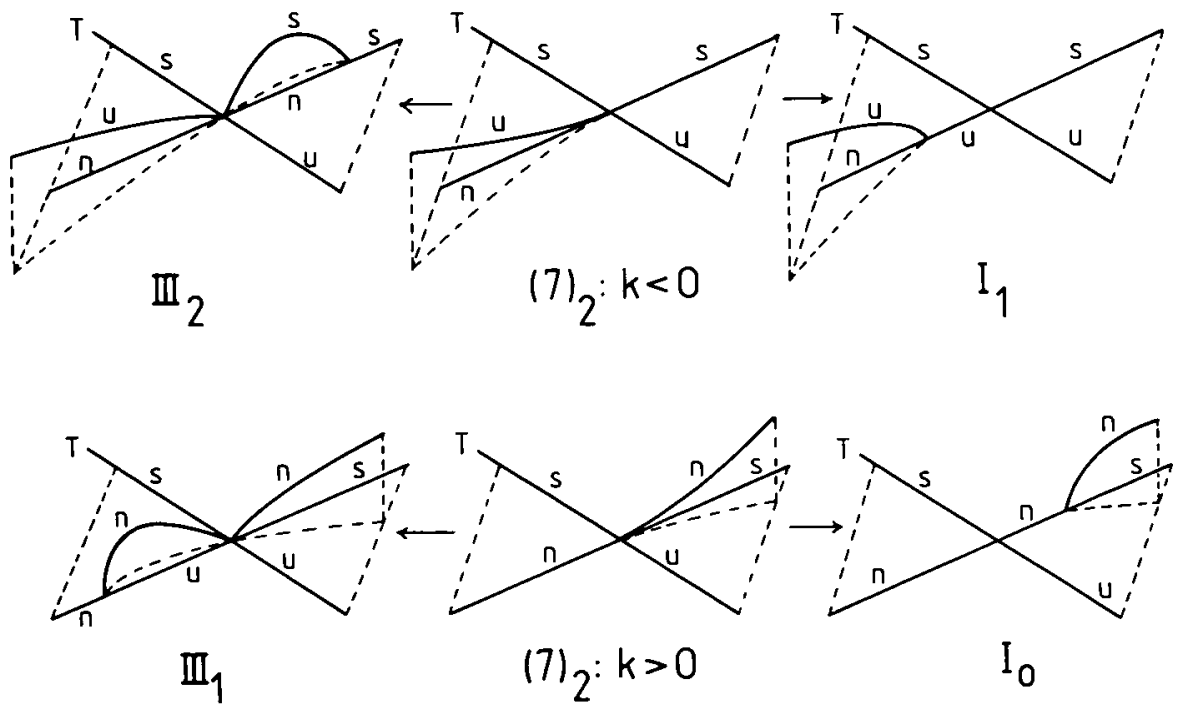

Figure 17. Type $(6)_{p}$ as subordinate bifurcation of $(9)_{31},(8)_{221}$ and $(7)_{2}$. 


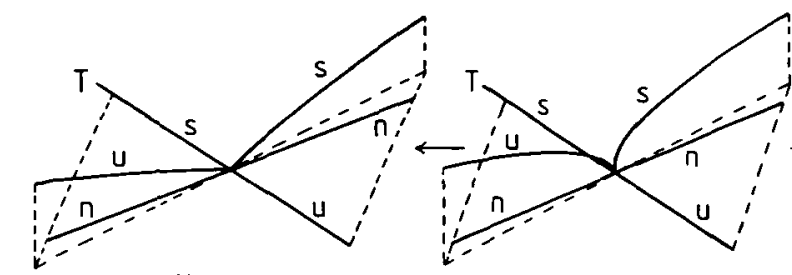

$\mathbb{I I}_{2}$
DIII

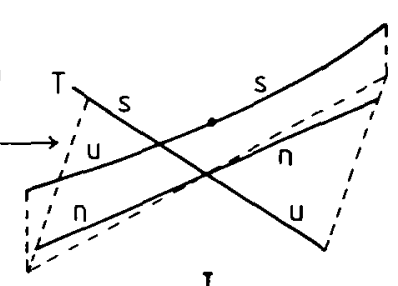

Io

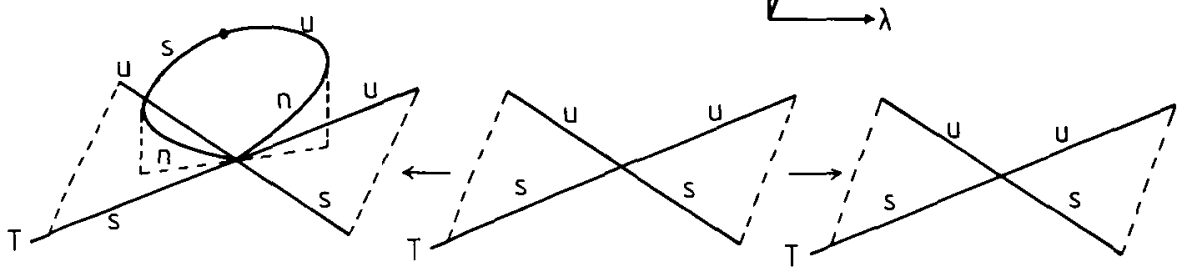

III $_{1}$

DI
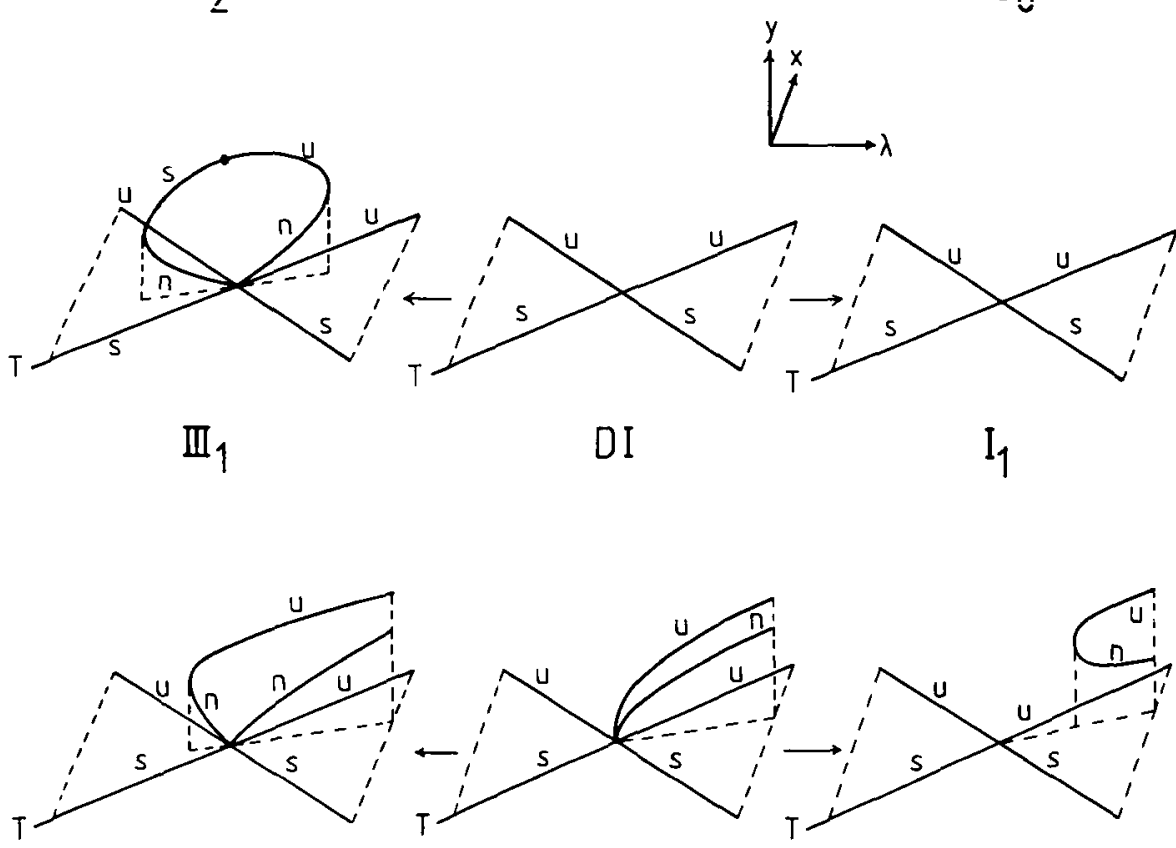

$\mathbb{I I}_{1}$

DI

$\mathrm{I}_{1}$

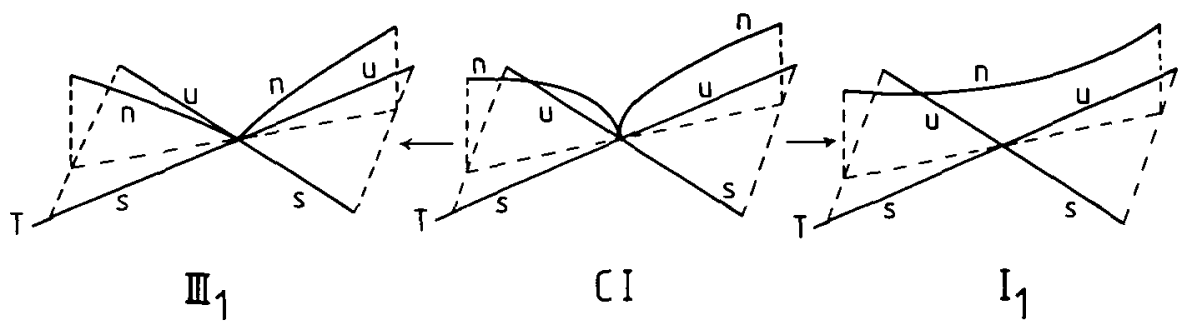

Figure 18. Type (6) $)_{\rho}$ as subordinate bifurcation of those modal cases of $(6 a)_{\rho, \kappa}$ which occur in the Brusselator. 


\section{Acknowledgement}

Fruitful discussions with Prof. W. Güttinger and Drs. D. Armbruster and F. J. Wright are gratefully acknowledged.

Work supported in part by the Stiftung Volkswagenwerk, Federal Republic of Germany.

\section{References}

[1] D. Armbruster, G. Dangelmayr and W. Güttinger, "Imperfection sensitivity of interacting Hopf and steady state bifurcations", Phys. D 16 (1985), 99-123.

[2] J. F. G. Auchmuty and G. Nicolis, "Bifurcation analysis of non-linear reaction diffusion equations I", Bull. Math. Biol. 37 (1975), 323-365.

[3] S. N. Chow and J. K. Hale, Methods of bifurcation theory (Springer, Berlin, 1982).

[4] G. Dangelmayr and D. Armbruster, "Classification of $Z(2)$-equivariant imperfect bifurcations with corank two", Proc. London Math. Soc. 46 (1983), 517-546.

[5] M. Golubitsky and D. Schaeffer, Singularittes and groups in bifurcation theory, Vol. I (Springer, New York, 1985).

[6] M. Golubitsky and D. Schaeffer, "Imperfect bifurcations in the presence of symmetry", Comm. Math. Phys. 67 (1979), 205-232.

[7] M. Golubitsky and W. F. Langford, "Classification and unfoldings of degenerate Hopf bifurcations", J. Differentıal Equations 41 (1981), 375-415.

[8] M. Herschkowitz-Kaufman, "Bifurcation analysis of non-linear reaction-diffusion equations II", Bull. Math. Biol. 37 (1975), 589-636.

[9] J. P. Keener, "Secondary bifurcation in non-linear diffusion reaction equations", Stud. Appl. Math. 55 (1976), 187-211.

[10] R. Lefever and I. Prigogine, "Symmetry breaking instabilities in dissipative systems", J. Chem. Phys. 48 (1968), 1695-1700.

[11] D. Schaeffer and M. Golubitsky, "Bifurcation analysis near a double degenerate eigenvalue of a model chemical reaction", Arch. Rational Mech. Anal. 75 (1981), 315-347.

[12] M. M. Vainberg and. V. A. Trenogin, Theory of branching of solutions of nonlinear equations (Noordhoff, Leyden, 1974).

\section{Appendix A}

The expressions for $p_{1}, p_{2}$ etc. occuring on the right-hand side of $(6.16 \mathrm{a}-\mathrm{c})$ are:

$$
\begin{aligned}
p_{1}= & -\frac{192(k+1)^{13}(2 k+1)\left(\mu_{1}+\mu_{2}\right)\left(144 \mu_{2}^{2}-23 \mu_{1} \mu_{2}-\mu_{1}^{2}\right)}{k^{14}\left(4 \mu_{2}-\mu_{1}\right)^{3}\left(9 \mu_{2}-\mu_{1}\right)\left(16 \mu_{2}-\mu_{1}\right)} \\
& -\frac{3 \pi^{2}(2 k+1)(k+1)^{7}\left(\mu_{1}+\mu_{2}\right)}{32 k^{14}\left(4 \mu_{1}-\mu_{2}\right)\left(4 \mu_{2}-\mu_{1}\right)}
\end{aligned}
$$




$$
\begin{gathered}
p_{1}=1548 \pi^{2}-\frac{2^{14} \cdot 11089}{55125}=11982.3 \\
p_{2}=\frac{192(k+1)^{13}(2 k+1)^{2}\left(\mu_{1}+\mu_{2}\right)}{k^{16}\left(4 \mu_{2}-\mu_{1}\right)^{3}} \\
q_{1}=\frac{\pi^{2}(2 k+1)(k+1)^{5}\left(3 \mu_{2}^{3}-4 \mu_{1}^{3}-15 \mu_{1}^{2} \mu_{2}-2 \mu_{1} \mu_{2}^{2}\right)}{2 k^{10}\left(4 \mu_{2}-\mu_{1}\right)\left(4 \mu_{1}-\mu_{2}\right)} \\
\quad+\frac{256(k+1)^{11}(2 k+1)\left(\mu_{1}+\mu_{2}\right)}{k^{12}\left(4 \mu_{2}-\mu_{1}\right)^{3}} \\
q_{1}=\frac{688128}{375}-\frac{1392 \pi^{2}}{5}=-912.7 \\
r_{1}=-\frac{2 \pi^{2}(2 k+1)^{2}(k+1)^{3}}{3\left(4 \mu_{2}-\mu_{1}\right) k^{6}} .
\end{gathered}
$$

\section{Appendix B}

Proof of Proposition 1 (Subsection 6.2). The first statement in Proposition 1 is obvious from the last expression in Appendix A. In order to prove that $q<0$ if $\theta>\bar{\theta}$ we need to show that $q_{1}$ is negative. For $k=1$ this is clear, so let $k \neq 1$. Write $q_{1}$ in the form $q_{1}=-q_{2} Q$ where

$$
q_{2}=\frac{(k+1)^{5}(2 k+1)}{\left(4 \mu_{2}-\mu_{1}\right)^{3}\left(4 \mu_{1}-\mu_{2}\right) k^{12}}>0,
$$

and $Q$ is the polynomial,

$$
\begin{aligned}
Q= & 81 \pi^{2} k^{12}+522 \pi^{2} k^{11}+\left(1191 \pi^{2}-1536\right) k^{10} \\
& -\left(9728-790 \pi^{2}\right) k^{9}-\left(25600+2899 \pi^{2} / 2\right) k^{8} \\
& -\left(34304+3817 \pi^{2}\right) k^{7}-\left(19200+8273 \pi^{2} / 2\right) k^{6} \\
& -\left(2608 \pi^{2}-10240\right) k^{5}+\left(27392-964 \pi^{2}\right) k^{4} \\
& +\left(23040-176 \pi^{2}\right) k^{3}+\left(10496-8 \pi^{2}\right) k^{2} \\
& +2560 k+256 .
\end{aligned}
$$

The coefficients multiplying the power $k^{n}$ in $Q$ are denoted by $a_{n}$. We have to show that $Q>0$ for all odd $k \neq 1$. 
(a) Let $k>0$. Then numerical estimates of the coefficients $a_{j}$ show that

$$
\begin{aligned}
& a_{12} k^{12}+a_{9} k^{9}+a_{6} k^{6}>100 k^{6}\left(7 k^{6}-20 k^{3}-700\right) \\
& a_{11} k^{11}+a_{8} k^{8}+a_{5} k^{5}>10^{3} k^{5}\left(5 k^{6}-40 k^{3}-16\right) \\
& a_{10} k^{10}+a_{7} k^{7}>10^{4} k^{7}\left(k^{3}-8\right) .
\end{aligned}
$$

The zeros of the right-hand sides of (B.1-3) are all $<3$. Since $a_{i}>0$ for $i \leqslant 4$ it follows that $Q>0$ if $k \geqslant 3$.

(b) If $k<0$ a similar estimate as in (a) shows that $a_{10} k^{10}+\cdots+a_{0}>0$ for $k \leqslant-3$ and $a_{12} k^{12}+a_{11} k^{11}>0$ for $k \leqslant-7$ which guarantees that $Q>0$ if $k \leqslant-7$. By substituting $k=-3$ and $k=-5$ into (B.1) we find that $Q>0$ also for these values of $k$. 